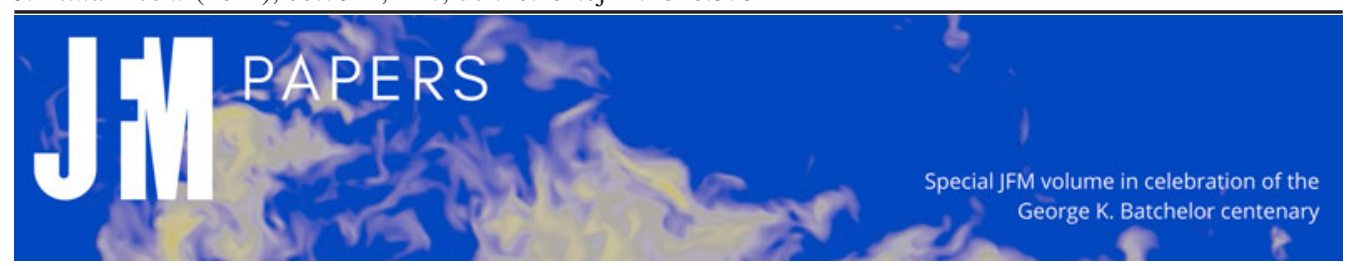

\title{
Near-wake structure of full-scale vertical-axis wind turbines
}

\author{
Nathaniel J. Wei ${ }^{1}$, Ian D. Brownstein ${ }^{2}$, Jennifer L. Cardona ${ }^{1}$, \\ Michael F. Howland ${ }^{1}$ and John O. Dabiri ${ }^{3}{ }^{\dagger}$ \\ ${ }^{1}$ Department of Mechanical Engineering, Stanford University, Stanford, CA 94305, USA \\ ${ }^{2}$ XFlow Energy Company, Seattle, WA 98108, USA \\ ${ }^{3}$ Graduate Aerospace Laboratories \& Mechanical Engineering, California Institute of Technology, \\ Pasadena, CA 91125, USA
}

(Received 20 December 2019; revised 30 June 2020; accepted 9 July 2020)

To design and optimize arrays of vertical-axis wind turbines (VAWTs) for maximal power density and minimal wake losses, a careful consideration of the inherently three-dimensional structure of the wakes of these turbines in real operating conditions is needed. Accordingly, a new volumetric particle-tracking velocimetry method was developed to measure three-dimensional flow fields around full-scale VAWTs in field conditions. Experiments were conducted at the Field Laboratory for Optimized Wind Energy (FLOWE) in Lancaster, CA, using six cameras and artificial snow as tracer particles. Velocity and vorticity measurements were obtained for a $2 \mathrm{~kW}$ turbine with five straight blades and a $1 \mathrm{~kW}$ turbine with three helical blades, each at two distinct tip-speed ratios and at Reynolds numbers based on the rotor diameter $D$ between $1.26 \times 10^{6}$ and $1.81 \times 10^{6}$. A tilted wake was observed to be induced by the helical-bladed turbine. By considering the dynamics of vortex lines shed from the rotating blades, the tilted wake was connected to the geometry of the helical blades. Furthermore, the effects of the tilted wake on a streamwise horseshoe vortex induced by the rotation of the turbine were quantified. Lastly, the implications of this dynamics for the recovery of the wake were examined. This study thus establishes a fluid-mechanical connection between the geometric features of a VAWT and the salient three-dimensional flow characteristics of its near-wake region, which can potentially inform both the design of turbines and the arrangement of turbines into highly efficient arrays.

Key words: wakes, vortex dynamics

$†$ Email address for correspondence: jodabiri@caltech.edu 


\section{N.J. Wei and others}

\section{Introduction}

Wind turbines are becoming increasingly important contributors to global energy supplies, as they represent a low-carbon alternative to traditional power-generation technologies that rely on the combustion of fossil fuels. If wind power is to comprise a larger share of global energy production, the efficiency and power density of wind farms will need to be improved (Jacobson \& Archer 2012). The critical limitation of these large arrays is not the efficiency of individual wind turbines, which already operate at efficiencies approaching their theoretical maximum (Betz 1920), but rather the dynamics of wind-turbine wakes and its effects on downstream turbines (Stevens \& Meneveau 2017). The efficiency of the large-scale deployment of wind power thus depends largely on a more careful consideration of the wake dynamics of wind turbines.

Horizontal-axis wind turbines (HAWTs) typically have very long wakes that extend up to 20 turbine diameters $(D)$ downstream of the turbine itself (Vermeer, Sørensen \& Crespo 2003; Meyers \& Meneveau 2012; Hau 2013; Stevens \& Meneveau 2017), although wake persistence may vary with field conditions (e.g. Nygaard \& Newcombe 2018). HAWTs placed in closely packed arrays therefore generally incur significant losses from the wakes of upstream turbines (e.g. Barthelmie et al. 2007; Barthelmie \& Jensen 2010; Barthelmie et al. 2010). Vertical-axis wind-turbine (VAWT) wakes, by contrast, have been observed to recover their kinetic energy within 4 to $6 D$ downstream of the turbine, albeit with lower individual coefficients of thrust and power (Kinzel, Mulligan \& Dabiri 2012; Kinzel, Araya \& Dabiri 2015; Ryan et al. 2016). VAWTs can also be arranged in pairs, to capitalize on synergistic fluid interactions between the turbines (e.g. Rajagopalan, Rickerl \& Klimas 1990; Ahmadi-Baloutaki, Carriveau \& Ting 2016; Brownstein, Kinzel \& Dabiri 2016; Hezaveh et al. 2018; Brownstein, Wei \& Dabiri 2019). Taken together, these factors imply that arrays of VAWTs can potentially achieve power densities an order of magnitude higher than those of conventional wind farms (Dabiri 2011).

The dynamics of VAWT wakes is therefore relevant to the design of large-scale wind farms with higher energy densities, and accordingly has been analysed in several recent studies. The replenishment of momentum in both HAWT and VAWT wakes has been shown to be dependent on turbulent entrainment of fluid from above the turbine array, through modelling (Meneveau 2012; Luzzatto-Fegiz \& Caulfield 2018), simulations (Calaf, Meneveau \& Meyers 2010; Hezaveh \& Bou-Zeid 2018), wind-tunnel experiments (Chamorro \& Porté-Agel 2009; Cal et al. 2010) and field experiments (Kinzel et al. 2015). VAWTs also exhibit large-scale vortical structures in their wakes that may further augment wake recovery. This vortex dynamics has been observed in scale-model studies of varying geometric fidelity, from rotating circular cylinders (Craig, Dabiri \& Koseff 2016) to complete rotors (e.g. Tescione et al. 2014; Brownstein et al. 2019). The inherently three-dimensional nature of these vortical structures, coupled with the high Reynolds numbers of operational VAWTs, complicates experimental and numerical studies of the dynamics of VAWT wakes.

Accordingly, numerical simulations with varying levels of complexity have been applied to study VAWT wakes. For studies of wake interactions within arrays, two-dimensional (2-D) Reynolds-averaged Navier-Stokes (RANS) simulations have often been employed (e.g. Bremseth \& Duraisamy 2016; Zanforlin \& Nishino 2016). Large-eddy simulation (LES) studies have generally used actuator-line models to approximate the effects of the individual blades on the flow (e.g. Shamsoddin \& Porté-Agel 2014, 2016; Abkar \& Dabiri 2017; Abkar 2018; Hezaveh \& Bou-Zeid 2018). Posa et al. (2016) and Posa \& Balaras (2018) were able to resolve the unsteady vortex shedding of individual blades in the spanwise component of vorticity using LES with periodic boundary conditions in the 
spanwise direction, which meant that tip-vortex shedding was not captured. More recently, Villeneuve, Boudreau \& Dumas (2020) used delayed detached-eddy simulations and a fully three-dimensional turbine model in a rotating overset mesh to study the effects of end plates on VAWT wakes, resolving 3-D vortex shedding and the wake dynamics up to $10 \mathrm{D}$ into the wake. Numerical simulations have thus continued to improve in their capacity to resolve the salient dynamics in the wakes of VAWTs.

Laboratory- and field-scale experiments have been extensively employed to characterize the wake dynamics of HAWTs over a large range of configurations and inflow conditions (e.g. Chamorro \& Porté-Agel 2009; Bastankhah \& Porté-Agel 2017; Schottler et al. 2017; Bartl et al. 2018), and similar laboratory-scale experiments have proved invaluable for the analysis of the wake structures of VAWTs as well. Experiments with VAWTs, however, have generally been limited to planar measurements in the laboratory (e.g. Brochier, Fraunie \& Beguierj 1986; Ferreira et al. 2009; Battisti et al. 2011). The deployment of stereoscopic particle-image velocimetry (stereo-PIV) has allowed some three-dimensional effects to be captured (Tescione et al. 2014; Rolin \& Porté-Agel 2015, 2018), as has the use of planar PIV with multiple imaging planes (Parker \& Leftwich 2016; Araya, Colonius \& Dabiri 2017; Parker, Araya \& Leftwich 2017). These planar techniques have been successful in identifying characteristic vortex phenomena, such as dynamic stall on turbine blades (Ferreira et al. 2009; Buchner et al. 2015; Dunne \& McKeon 2015; Buchner et al. 2018) and tip-vortex shedding from the ends of individual blades (Hofemann et al. 2008; Tescione et al. 2014). Generally, however, it is difficult to compute all three components of vorticity or the circulation of vortical structures with purely planar measurements. The analysis of the three-dimensional character of vortical structures in the wake is therefore greatly facilitated by fully three-dimensional flow-field measurements. Such experiments have only recently been carried out in laboratory settings. Using tomographic PIV, Caridi et al. (2016) resolved the three-dimensional structure of tip vortices shed by a VAWT blade within a small measurement volume with a maximum dimension of 5.5 blade chord lengths. Ryan et al. (2016) obtained 3-D time-averaged velocity and vorticity measurements of the full wake of a model VAWT using magnetic-resonance velocimetry. Most recently, Brownstein et al. (2019) used 3-D particle-tracking velocimetry (PTV) to obtain time-averaged measurements of the wakes of isolated and paired VAWTs. These studies were all carried out at laboratory-scale Reynolds numbers, which fell between one and two orders of magnitude below those typical of operational VAWTs. Miller et al. (2018a) attained Reynolds numbers up to $\operatorname{Re}_{D}=5 \times 10^{6}$ using a compressed-air wind tunnel, and their measurements of the coefficients of power demonstrated a Reynolds number invariance for $\operatorname{Re}_{D}>1.5 \times 10^{6}$. These power measurements suggest that Reynolds numbers of the order of $10^{6}$ may be required to fully capture the wake dynamics of field-scale turbines. As an alternative to laboratory experiments at lower $\operatorname{Re}_{D}$, experiments in field conditions at full scale are possible, but these have been limited to pointwise anemometry (Kinzel et al. 2012, 2015) or 2-D planar velocity measurements (Hong et al. 2014). Thus, for the validation and extension of existing experimental work on the wake dynamics of VAWTs, 3-D flow measurements around full-scale VAWTs in field conditions are desirable.

The additional benefit of 3-D flow measurements is that they enable the effects of complex turbine geometries on wake structures to be investigated. This is particularly useful for VAWTs, since several distinct geometric variations exist. Because of the planar constraints of most experimental and numerical studies, VAWTs with straight blades and constant spanwise cross-sections have primarily been studied due to their symmetry and simplicity of construction. However, there exist several VAWT designs that incorporate curved blades. Large-scale Darrieus-type turbines have blades that are bowed outward 


\section{N.J. Wei and others}

along the span, and these have historically reached larger sizes and power-generation capacities than straight-bladed turbines (Möllerström et al. 2019). Similarly, many modern VAWTs have helical blades that twist around the axis of rotation, following the design of the Gorlov Helical Turbine (Gorlov 1995), to reduce fatigue from unsteady loads on the turbine blades. Relatively few studies have investigated the flow physics of these helical-bladed VAWTs in any kind of detail. Schuerich \& Brown (2011) used a vorticity-transport model for this purpose, and Cheng et al. (2017) approached the problem using unsteady RANS, 2-D LES and wind-tunnel experiments. Aliferis et al. (2019) studied the 2-D planar wake structure of a Savonius-type VAWT with helical blades using a Cobra probe on a traverse. Lastly, Ouro et al. (2019) analysed turbulence quantities in the wake of a helical-bladed VAWT in a water channel using pointwise velocity measurements from an anemometer on a traverse. A full treatment of the effects of the helical blades on the three-dimensional vorticity fields and corresponding vortical structures in the wake has yet to be undertaken.

Thus, the purpose of this work is to study the three-dimensional flow features of operational VAWTs in the field. The work revolves around two primary contributions: the development of a field-deployed 3-D PTV measurement system, and the analysis of topological and dynamical characteristics of vortical structures in the wakes of full-scale VAWTs. Firstly, the characterization of the artificial-snow-based technique for obtaining three-dimensional, three-component measurements of velocity and vorticity in field experiments around full-scale VAWTs will be documented $(\S 2)$. Experiments with two VAWTs, one with straight blades and one with helical blades and each at two tip-speed ratios, will then be outlined. Velocity and vorticity fields from these experiments will be presented, and a difference in the three-dimensional structure of the wake between the two types of turbines will be identified ( $\S 3.1$ and 3.2). This tilted-wake behaviour will be analysed further, to establish a connection between blade geometry and wake topology (§3.3). The results from these analyses shed light on the dynamics that governs the near wake of VAWTs (up to $\sim 3 D$ downstream, cf. Araya et al. 2017), and implications of these findings for future studies and for the design and optimization of VAWT wind farms will be discussed ( $\$ \S 3.4$ and 3.5). This work represents the first full-scale, fully three-dimensional flow-field study on operational VAWTs in field conditions, and therefore provides fundamental insights into the wake dynamics at high Reynolds numbers and the fluid mechanics of wind energy.

\section{Experimental methods}

In this section, the set-up of the field experiments is outlined, and a novel technique for 3-D PTV measurements in field conditions is introduced. The experimental procedure is discussed, and the post-processing steps for computing velocity and vorticity fields are described. Additional details and characterizations of the measurement system are given in appendices $\mathrm{A}$ and $\mathrm{B}$.

\subsection{Field site and turbine characterization}

Experiments were carried out during the nights of 9-11 August 2018 at the Field Laboratory for Optimized Wind Energy (FLOWE), located on a flat, arid segment of land near Lancaster, California, USA. Details regarding the geography of the site are provided by Kinzel et al. (2012). Wind conditions at the site were measured with an anemometer (First Class, Thies Clima) and a wind vane (Model 024A, Met One), which recorded data at $1 \mathrm{~Hz}$ with accuracies of $\pm 3 \%$ and $\pm 5^{\circ}$, respectively. These were mounted on a 
(a)

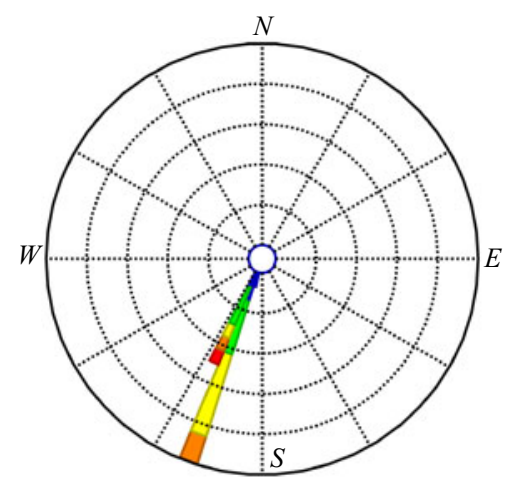

Wind speeds $\left(\mathrm{m} \mathrm{s}^{-1}\right)$ $11 \leq U_{\infty}<12$

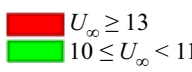

$12 \leq U_{\infty}<13$
$9 \leq U_{\infty}<10$ (b)
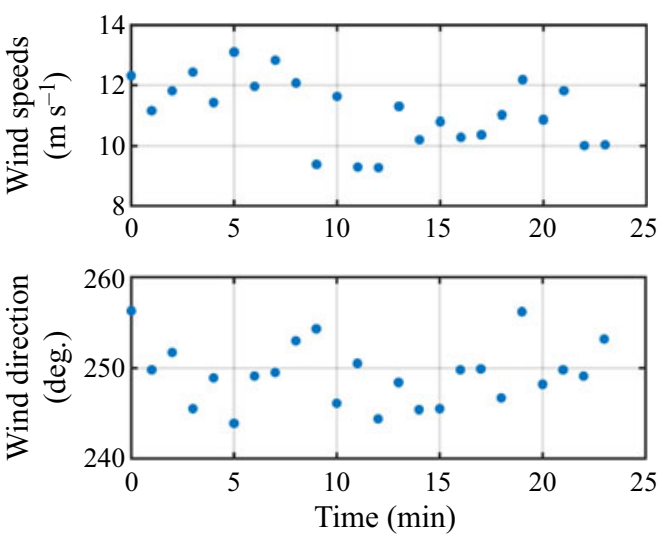

Figure 1. (a) Wind rose for conditions during experiments (9-11 August 2018). The plotted wind speeds and directions are those recorded by the tower-mounted anemometer, located $10 \mathrm{~m}$ above the ground, and have been binned in ten minute averages by $1 \mathrm{~ms}^{-1}$ and $5^{\circ}$, respectively. $(b)$ Wind speeds and directions, from a single experiment (three eight minute data sets), binned in one minute averages.

meteorological tower (Model M-10M, Aluma Tower Co.) at a height of $10 \mathrm{~m}$ above the ground. The tower also recorded air temperature, which was used to interpolate air density from a density-temperature table. A datalogger (CR1000, Campbell Scientific) recorded these data at 1 and 10 minute intervals. The height difference between the anemometer and the VAWTs was corrected using a fit of an atmospheric boundary-layer profile to data collected previously at the site at multiple heights (cf. Kinzel et al. 2012). The correction resulted in a $3 \%$ change in the free-stream velocity, which compared more favourably with the particle-based flow-field measurements than the uncorrected readings. The measured wind conditions at the site during experiments were uniform in both magnitude and direction: the wind speed was $11.01 \pm 1.36 \mathrm{~ms}^{-1}$, and the wind direction was from the southwest at $248 \pm 3^{\circ}$. These statistics were calculated from sensor data that had been averaged by the datalogger into ten minute readouts, and are summarized in the wind rose shown in figure $1(a)$.

For these experiments, two types of VAWTs were employed. A 1-kW, three-bladed VAWT with helical blades, built by Urban Green Energy (UGE), was compared with a 2-kW, five-bladed VAWT with straight blades from Wing Power Energy (WPE). The blades of both turbines had constant cross-sectional geometries. The blade twist of the helical-bladed turbine, representing the angle of twist with respect to the axis of rotation per unit length along the span of the turbine, was $\tau=0.694 \mathrm{rad} \mathrm{m}^{-1}$. Photos and details of these two turbines, referred to in this work by their manufacturer's acronyms (UGE and WPE), are given in figure 2. Each turbine was tested at two different tip-speed ratios, defined as

$$
\lambda=\frac{\omega R}{U_{\infty}},
$$

where $\omega$ is the rotation rate of the turbine $\left(\mathrm{rad} \mathrm{s}^{-1}\right), R$ is the radius of the turbine $(\mathrm{m})$ and $U_{\infty}$ is the magnitude of the free-stream velocity $\left(\mathrm{ms}^{-1}\right)$. The turbines had different solidities, quantified as the ratio of the blade area to the swept area of the rotating blades. This was defined as

$$
\sigma=\frac{n c}{\pi D}
$$




\section{N.J. Wei and others}

(a)

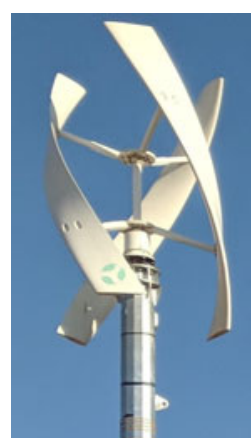

(c)

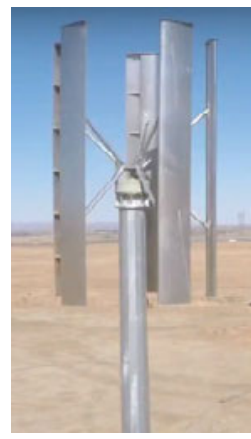

(b)

Manufacturer

Model

Rated power

Number of blades

Turbine radius

Turbine span

Blade chord

Solidity $(\sigma)$

(d)

\begin{tabular}{l|l}
\hline Manufacturer & Wing Power Energy \\
Model & N/A \\
Rated power & $2 \mathrm{~kW}$ \\
Number of blades & 5 \\
Turbine radius & $1.1 \mathrm{~m}$ \\
Turbine span & $3.7 \mathrm{~m}$ \\
Blade chord & $0.483 \mathrm{~m}$ \\
Solidity $(\sigma)$ & $0.349 \pm 0.020$
\end{tabular}

Figure 2. Photographs $(a, c)$ and specifications $(b, d)$ of the helical-bladed UGE turbine $(a, b)$ and the straight-bladed WPE turbine $(c, d)$. The blade twist of the UGE turbine is $\tau=0.694 \mathrm{rad} \mathrm{m}^{-1}$.

Case Identifier

Turbine

Wind Speed, $U_{\infty}\left(\mathrm{ms}^{-1}\right)$

Duration, $T$ (s)

Duration, $T^{*}=T\left(U_{\infty} / D\right)(-)$

Reynolds Number, $R e_{D} \times 10^{6}$

Tip-Speed Ratio, $\lambda$

Solidity, $\sigma$

$\begin{array}{cc}\Delta & \nabla \\ \text { UGE } & \text { UGE } \\ 11.15 \pm 1.37 & 11.39 \pm 1.25 \\ 1393 & 1343 \\ 8627 & 8491 \\ 1.26 \pm 0.15 & 1.29 \pm 0.14 \\ 1.19 \pm 0.13 & 1.40 \pm 0.14 \\ 0.271 \pm 0.030 & 0.271 \pm 0.030\end{array}$

$12.76 \pm 1.54$

1453

8423

$1.81 \pm 0.22$

$0.96 \pm 0.10$

$0.349 \pm 0.020$

WPE

$10.10 \pm 1.29$

1393

6396

$1.43 \pm 0.18$

$1.20 \pm 0.16$

$0.349 \pm 0.020$

Table 1. Experimental parameters for the four test cases presented in this work. From the left, the first two experiments were carried out on 9 August 2018, the third on 10 August and the fourth on 11 August. The non-dimensional duration $T^{*}$ represents the number of convective time units $D / U_{\infty}$ captured by each experiment. Uncertainties from the average values represent one standard deviation over time.

where $n$ is the number of blades, $c$ is the chord length of each blade and $D$ is the turbine diameter. The parameters for the four experiments presented in this work are given in table 1.

The turbines were mounted on the same tower for experiments, setting the mid-span location of each at a height of $8.2 \mathrm{~m}$ above the ground. A Hall-effect sensor (Model 55505, Hamlin) on the tower measured the rotation rate of the WPE turbine by recording the blade passing frequency. This method could not be implemented with the UGE turbine due to its different construction. Therefore, the rotation rate of the UGE turbine was calculated from videos of the turbine in operation, taken at 120 frames per second with a CMOS camera 


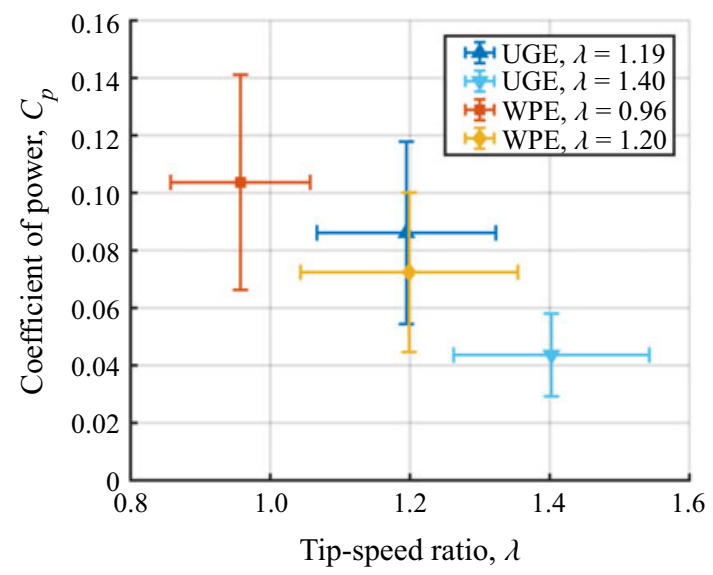

Figure 3. Coefficient of power as a function of tip-speed ratio for the four experiments outlined in table 1.

(Hero4, GoPro), by autocorrelating the pixel-intensity signal to establish a blade passing time. Electrical power outputs from the turbines were measured and recorded in 10-minute intervals using a second datalogger (CR1000, Campbell Scientific). The coefficient of power was then calculated as

$$
C_{p}=\frac{P}{\frac{1}{2} \rho S D U_{\infty}{ }^{3}},
$$

where $P$ is the power produced by the turbine, $\rho$ is the density of air and $S$ is the turbine span. The computed coefficients of power of the two turbines for each of the tested tip-speed ratios are shown in figure 3 . The $C_{p}$ values for both turbines agree with measurements from previous experiments at the FLOWE field site reported by Miller et al. (2018a) that suggested that the optimal tip-speed ratio for maximizing $C_{p}$ was of the order of $\lambda \approx 1$ for the WPE turbine. This operating tip-speed ratio is low compared to those of larger-scale VAWTs with lower solidities (cf. Möllerström et al. 2019), but is consistent with those of turbines of the same power-production class (e.g. Han et al. 2018). The results also agree with the findings of other studies that the optimal tip-speed ratio for power production decreases with increasing solidity (Miller et al. 2018b; Rezaeiha, Montazeri \& Blocken 2018).

\subsection{Particle-tracking velocimetry}

A new method for volumetric flow measurements in field conditions was developed to obtain three-dimensional velocity measurements in a large measurement volume encapsulating the near wake of full-scale turbines. Because of the arid climate at the field site, natural precipitation could not be relied on to populate the required measurement volume with seeding particles (cf. Hong et al. 2014). Therefore, to quantify the flow around the turbines, artificial snow particles were used as seeding particles for the flow. These were produced by four snow machines (Silent Storm DMX, Ultratec Special Effects) that were suspended by cables from two poles approximately four turbine diameters $(D)$ upstream of the turbine tower. The machines could be raised to different heights with respect to the turbine, to adjust the distribution of particles in the measurement volume. The particles were illuminated by two construction floodlights (MLT3060, Magnum), so that their images contrasted the night sky. Six CMOS video cameras (Hero4, GoPro) 


\section{N.J. Wei and others}

(a)

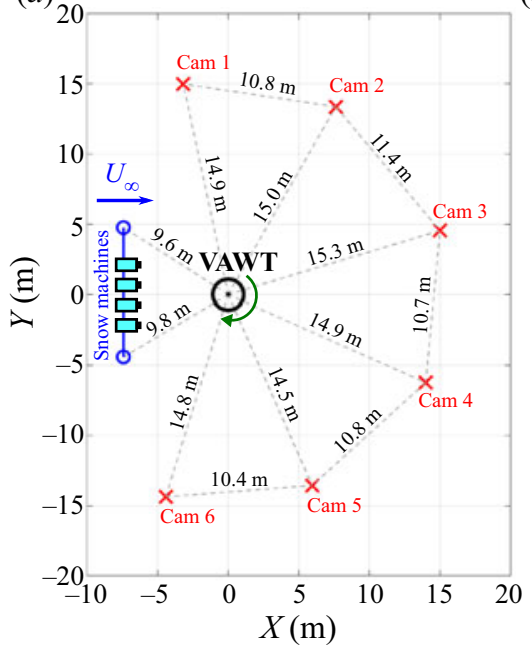

(b)

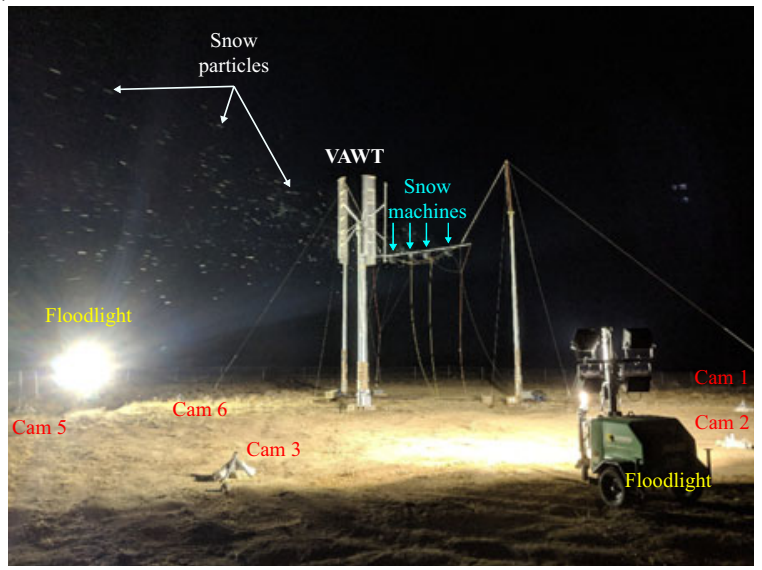

Figure 4. Schematic $(a)$ and photograph $(b)$ of the field experiment. The snow machines in $(a)$ are not drawn to scale. The video cameras are labelled as Cam 1 through Cam 6. The direction of rotation for the turbine in the diagram is clockwise, and the $Z$-coordinate points vertically upward from the ground. The WPE turbine $(S=3.7 \mathrm{~m})$ is shown in the photo. The artificial snow particles are visible moving with the flow toward the left of the frame.

were mounted on frames and installed in a semicircle on the ground to capture the particles in the turbine wake from several different angles. The layout of the entire experiment (excluding the upstream meteorological tower) is shown in figure 4. The low seeding density and high visibility of the particles under these conditions meant that unambiguous particle trajectories could be extracted from the camera images, making PTV a natural choice for obtaining three-dimensional, three-component volumetric velocity measurements.

The degree to which the artificial snow particles follow the flow was an important factor for the accuracy of the PTV measurements, and their aerodynamic characteristics were accordingly considered in detail. The particles were composed of an air-filled soap foam with an average effective diameter of $d_{p}=11.2 \pm 4.2 \mathrm{~mm}$ and an average density of $\rho=6.57 \pm 0.32 \mathrm{~kg} \mathrm{~m}^{-3}$. Since the particles were relatively large and non-spherical, experiments were conducted in laboratory conditions to establish their aerodynamic characteristics. A detailed description of the experiments, results and analyses regarding particle response and resulting experimental error is given in appendix A. By releasing the particles into a wind tunnel as a jet in cross-flow and tracking them using 3-D PTV with four cameras, the particle-response time scale $\tau_{p}$ and slip velocity $V_{s}$ were computed. Comparing $\tau_{p}$ with the relevant flow time scale, $\tau_{f}=D / U_{\infty}$, yielded a particle Stokes number of $S k=\tau_{p} / \tau_{f} \approx 0.23$. The worst-case slip velocities for the field experiments were estimated to be $V_{s} \lesssim 0.170 \mathrm{~ms}^{-1}$, or less than $2 \%$ of the average wind speed. Therefore, the particles were found to follow the flow with sufficient accuracy to resolve the large-scale structures encountered in VAWT wakes in the field.

To ensure that the entire measurement domain was sampled with sufficient numbers of particles, multiple iterations of each experimental case were conducted, focusing on different regions of the measurement volume. This was accomplished by raising the snow machines to three distinct heights with respect to the turbine: at the turbine mid-span, above the turbine mid-span and at the top of the turbine (approximately 8,9 and $10 \mathrm{~m}$ 
above the ground, respectively). Vector fields obtained from the data for each case were combined, so that each experiment listed in table 1 represents the combination of three separate recording periods. The effects of time averaging are further discussed in $§ 2.4$.

The six video cameras were arranged in a semicircle around the near-wake region of the turbines, up to 7 to $8 D$ downstream of the turbine. They recorded video at 120 frames per second and a resolution of $1920 \times 1080$ pixels, with an exposure time of 1/480 s and an image sensitivity (ISO) of 6400. The test cases outlined in table 1 thus represent between 161000 and 175000 images per camera of the measurement volume. Because the cameras were all positioned on the downstream side of the turbine, some flow regions adjacent to the turbine were masked by the turbine itself, and thus could not be measured. This, however, did not affect the measurement of the wake dynamics downstream of the turbine. The total measurement volume was approximately $10 \mathrm{~m} \times 7 \mathrm{~m} \times 7 \mathrm{~m}$, extending up to $2 D$ upstream of the turbine and at least $3 D$ downstream into the wake.

To achieve the 3-D reconstruction of the artificial snow particles in physical space from the 2-D camera images, a wand-based calibration procedure following that of Theriault et al. (2014) was carried out. A description of the procedure and its precision is included in appendix B.1. The two calibrations collected at the field site resulted in reconstruction errors of distances between cameras of $0.74 \pm 0.39 \%$ and $0.83 \pm 0.41 \%$, and reconstruction errors in the spans of the turbines of $0.21 \%$ and $0.32 \%$. The calibrations therefore allowed particle positions to be triangulated accurately in physical space.

\subsection{Experimental procedure}

The collection of data for the field experiments was undertaken as follows. The rotation rate of the turbine was controlled via electrical loading to change the tip-speed ratio between experiments. The snow machines were hoisted to the desired height relative to the turbine, and artificial snow particles were advected through the measurement domain by the ambient wind. All six cameras were then initiated to record 420 to 560 seconds of video. Wind speed and direction measurements from the meteorological tower were averaged and recorded in one minute bins over the duration of the recording period. The procedure was then repeated for the three different snow-machine heights listed in $\S 2.2$, corresponding to three data sets in total for each experimental case.

\subsection{Data processing and analysis}

The procedure and algorithms used to obtain accurate time-averaged velocity fields from the raw camera images are presented in detail in appendix B.2, along with an analysis of the statistical convergence of the averaged data. An overview of the procedure is given here.

Particles were isolated in the raw images through background subtraction and masking. Because the cameras were not synchronized via their hardware, the images from the camera views were temporally aligned using an LED band (RGBW LED strip, Supernight) that was mounted on the turbine tower below the turbine blades and flashed at one minute intervals. Particles were then identified in the synchronized images by thresholding based on pixel intensities. The particles were mapped to 3-D locations in physical space using epipolar geometry (Hartley \& Zisserman 2003). A multi-frame predictive-tracking algorithm developed by Ouellette, Xu \& Bodenschatz (2006) and Xu (2008) computed Lagrangian particle trajectories and velocities from these locations. Velocity data recorded with the snow machines set at different heights were combined into a single unstructured volume of instantaneous velocity vectors. This field was averaged into discrete cubic voxels 


\section{N.J. Wei and others}

with side lengths of $25 \mathrm{~cm}$. The standard deviation of the velocity magnitude over all vectors in these voxels was below $5 \%$ of the average value for over $50 \%$ of the voxels in the measurement domain. This quantity can be interpreted as an analogue for measurement precision, though some variation due to turbulence across vectors within the voxels was expected. In the wake of the turbine, the volume of interest for the analyses presented in this work, $87 \%$ of voxels had standard deviations below $5 \%$, with $58 \%$ having values below $2 \%$. The best-case precision for highly sampled voxels was below $1 \%$. A more thorough account of these statistical-convergence studies is provided in appendix B.2. The velocity fields were finally filtered to enforce a zero-divergence condition (Schiavazzi et al. 2014) and were used to compute vorticity fields.

It is important to briefly consider the effect of time averaging, which was inherent to this PTV method. Temporal averaging removed the presence of turbulence fluctuations in the data, and thus the resulting velocity and vorticity fields necessarily contained only flow phenomena that were present in the mean flow. Conjectures regarding the effects of turbulence fluctuations on momentum transfer into the wake are therefore not possible based on these measurements. These effects are expected to dominate the far wake, whereas the near wake is characterized by large-scale vortical structures. Therefore, for the purposes of this study, it was deemed acceptable to forgo the resolution of turbulence fluctuations. Similarly, unsteady vortex dynamics was not resolved in these experiments. However, the effects of vortex dynamics occurring at large scales can still be observed in the time-averaged flow fields, as will be shown in the following section. It is thus possible to infer connections between the time-averaged results of these experiments and the underlying unsteady dynamics observed in previous studies (e.g. Battisti et al. 2011; Tescione et al. 2014; Parker \& Leftwich 2016; Araya et al. 2017; Rolin \& Porté-Agel 2018).

\section{Experimental results}

In this section, the results of the field experiments described in the previous section are presented and further analysed. Firstly, the velocity fields for the four experimental cases given in table 1 are shown to highlight three-dimensional flow features. Next, the vorticity fields for these cases are presented, and a tilted wake is observed for the helical-bladed turbine. The tilted wake is then analysed further, and a connection between turbine-blade geometry and wake tilt is established. Finally, the dynamics of the identified vortical structures is considered, and the implications of the results for arrays of VAWTs are outlined.

\subsection{Velocity fields}

Velocity fields for the time-averaged streamwise-velocity component $U$ on three orthogonal planar cross-sections are given in figures 5 and 6 for the helical-bladed (UGE) and straight-bladed (WPE) turbines at tip-speed ratios of $\lambda=1.19$ and $\lambda=1.20$, respectively. The wake structure was not observed to change significantly with the changes in $\lambda$ achieved in these experiments. A more detailed analysis of the wake velocity fields for both turbines, including comparisons with previous wake studies at lower $R e_{D}$, is provided in appendix C.1. As this study seeks to ascertain the effects of turbine-blade geometry on the 3-D structure of the near wake, two key differences between the velocity fields of the two turbines are highlighted.

Firstly, in $Y Z$ cross-sections of $U$ downstream of the turbine, topological differences were evident in the wake region, where the local streamwise velocity fell below the free-stream velocity. This region was observed to tilt in the clockwise direction when viewed from downstream in the case of the helical-bladed turbine (figure 5), while no 
(a) $Z / D=0$
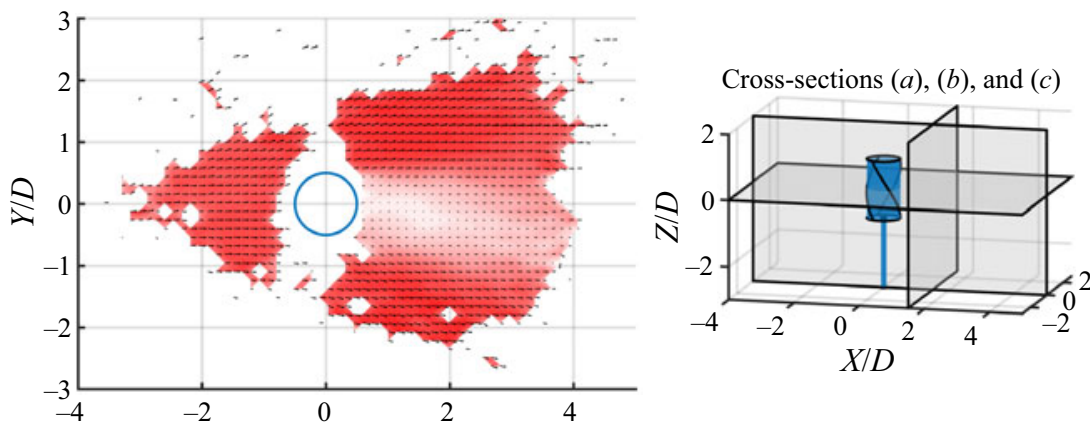

(b)

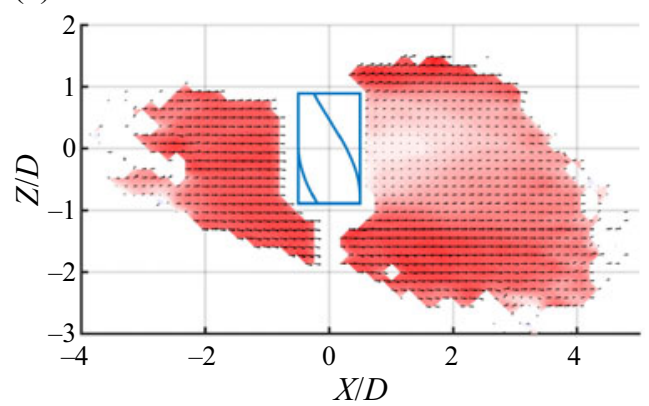

(c)

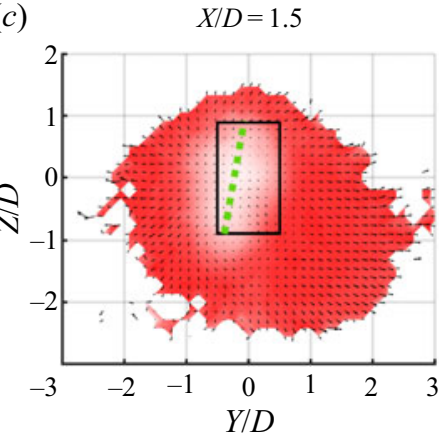

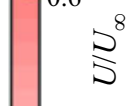

$-0.4$

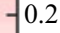

$-0$

Figure 5. Three orthogonal time-averaged planar fields of the streamwise velocity $U$ for the helical-bladed turbine, taken at $Z / D=0, Y / D=0$ and $X / D=1.5$ (counter-clockwise, from top left). A slight tilt from the vertical in the clockwise direction, shown by a fit to minima in the streamwise velocity (dashed green line), is visible in the velocity-deficit region in the $Y Z$ cross-section.

such tilt was observed in the case of the straight-bladed turbine (figure 6). This tilted-wake behaviour will be analysed in detail in $\S 3.3$, and it will be shown that this topological difference was a consequence of the blade shape of the helical-bladed turbine.

Secondly, a slice of the time-averaged vertical-velocity component $W$ through the central axis of the turbine further implied the existence of a more complex three-dimensional dynamics in the wake of the helical-bladed turbine (figure 7). The vector field at $Y / D=0$ for the straight-bladed turbine showed a downward sweep of fluid from above the turbine and an upward sweep of fluid from below the turbine, as would be expected from a bluff body in cross-flow (figure $7 b$ ). The corresponding field for the helical-bladed turbine showed a very different scenario, in which a uniform central updraft was present (figure 7a). At planar slices of $Y / D$ on either side of $Y / D=0$, corresponding uniform downward motions of fluid were observed. These differences in the vertical-velocity fields implied that the 3-D structure of the helical-bladed turbine had an effect on the wake dynamics that could not be resolved simply by examining the velocity fields. A three-dimensional analysis of the vortical structures present in the wakes of these VAWTs is required to explain these observed differences. This will be provided in §3.2.

\subsection{Vortical structures and wake topology}

An analysis of the vortical structures in the streamwise direction $\left(\omega_{x}\right)$ demonstrates the importance of three-dimensional considerations to the wake dynamics of these VAWTs. 


\section{N.J. Wei and others}

(a)

$Z / D=0$

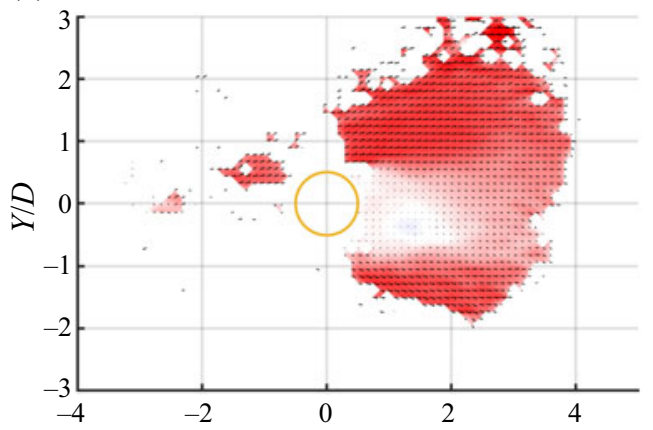

Cross-sections $(a),(b)$, and $(c)$

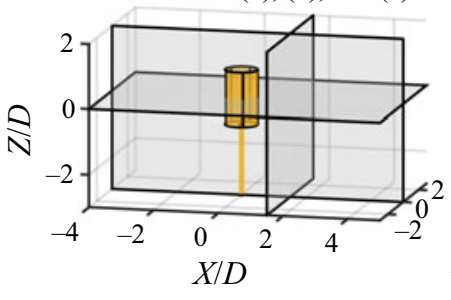

(b)

$Y / D=0$

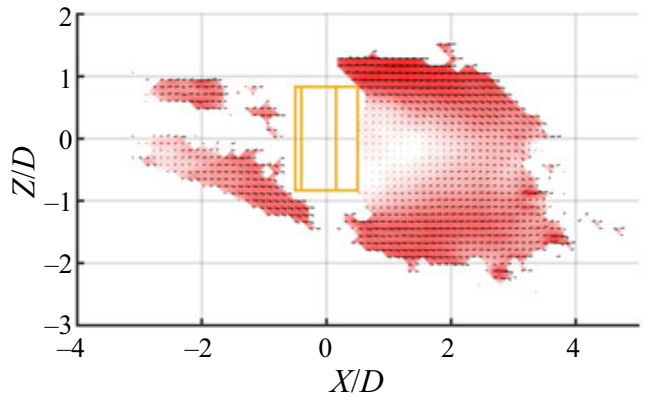

(c)

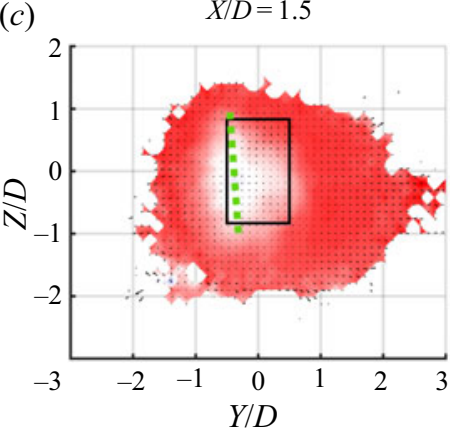

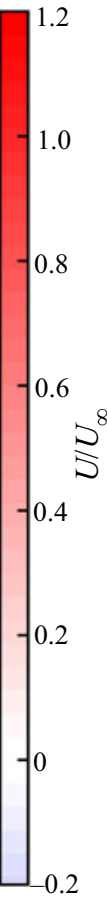

$-0.2$

Figure 6. Three orthogonal time-averaged planar fields of the streamwise velocity $U$ for the straight-bladed turbine, taken at $Z / D=0, Y / D=0$ and $X / D=1.5$ (counter-clockwise, from top left). In contrast to figure 5, no wake tilt is present in the $Y Z$ cross-section, as evidenced by the relatively vertical alignment of the fit to the wake profile (dashed green line).

(a)

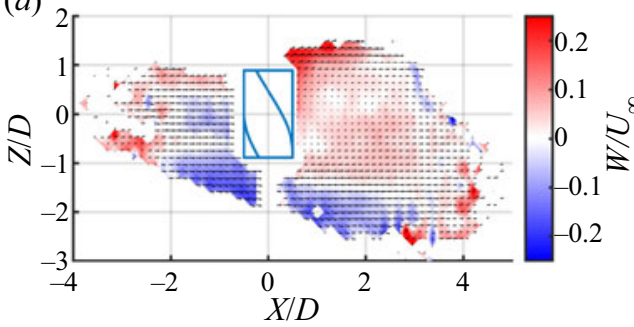

(b)

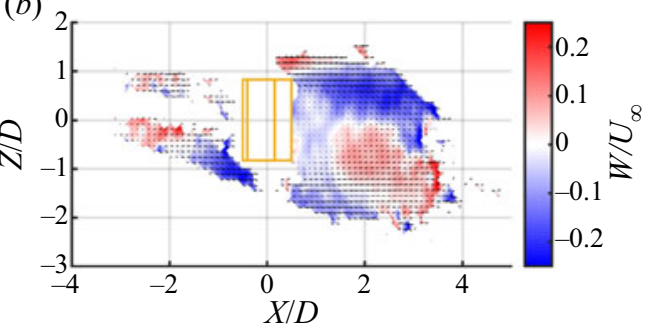

Figure 7. Time-averaged planar fields of the vertical velocity $W$ for $(a)$ the helical-bladed turbine at $\lambda=1.19$ and $(b)$ the straight-bladed turbine at $\lambda=1.20$, taken at $Y / D=0$. The wake of the straight-bladed turbine is characterized by symmetric sweeps of high-momentum fluid into the wake from above and below. In contrast, the wake of the helical-bladed turbine exhibits a uniform updraft at $Y / D=0$. This difference suggests that the helical blades have a pronounced three-dimensional effect on the wake structure.

Streamwise planar slices of $\omega_{x}$ are shown in figures 8 and 9. The structures visible in these plots comprised a horseshoe-shaped vortex induced by the rotation of the turbine, observed previously by Rolin \& Porté-Agel (2018) and Brownstein et al. (2019). While in the case of the straight-bladed turbine, the two branches of the vortex were symmetric about the mid-span of the turbine, the corresponding structure for the helical-bladed turbine was asymmetric. The offset of the upper branch with respect to the lower branch induced 

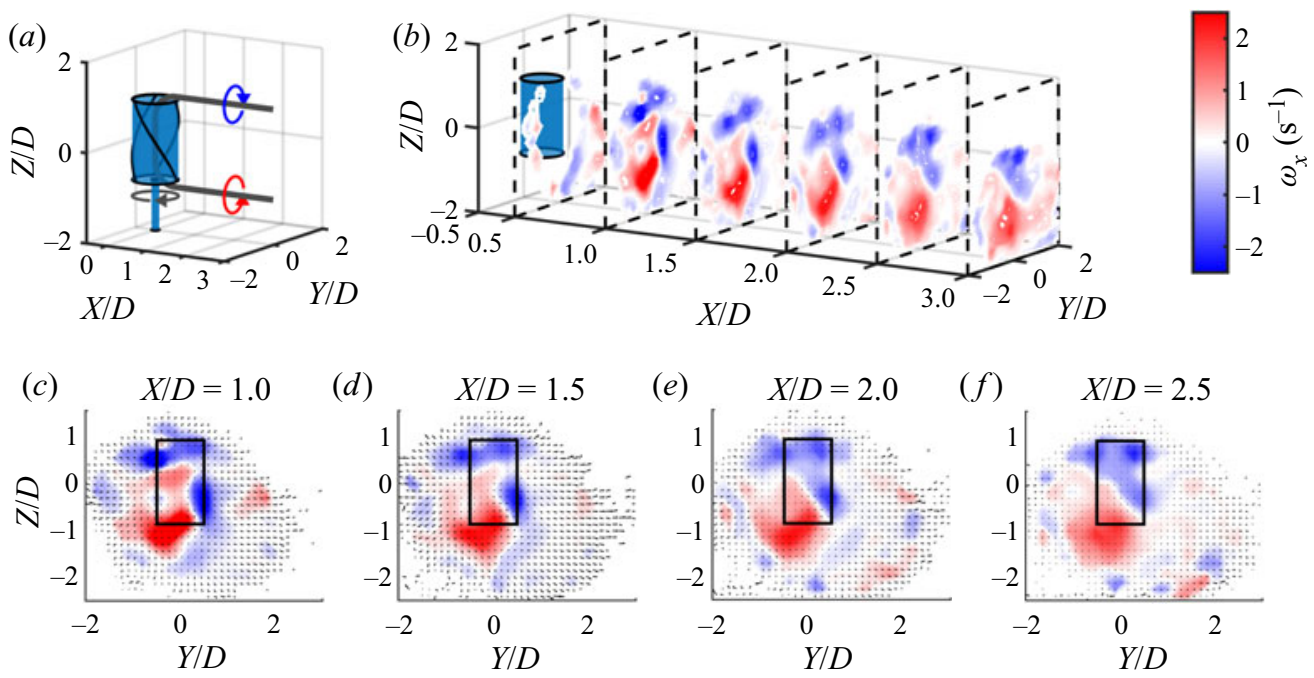

Figure 8. Streamwise slices of the streamwise vorticity $\omega_{x}$ in the case of the helical-bladed turbine for $\lambda=1.19$. The $X$-axis is stretched on $0.5 \leq X / D \leq 3$ to show the slices more clearly. These fields show marked asymmetry and a vertical misalignment in the two branches of the horseshoe vortex induced by the rotation of the turbine, compared to those shown in figure 9.

(a)

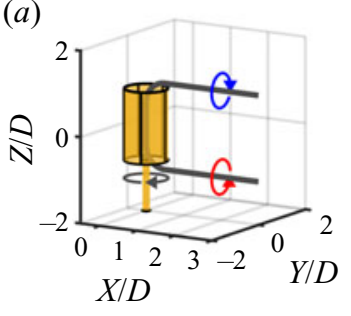

(b)

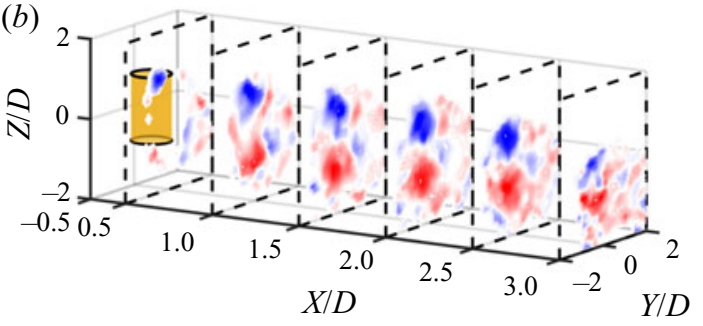

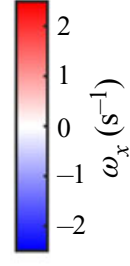

(e)

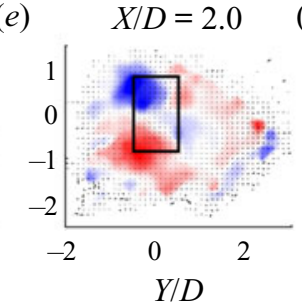

(f) $\quad X / D=2.5$

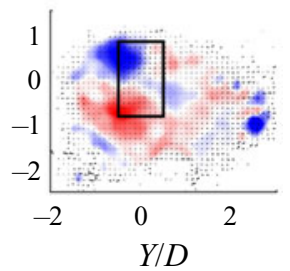

Figure 9. Streamwise slices of the streamwise vorticity $\omega_{x}$ in the case of the straight-bladed turbine for $\lambda=1.20$. The $X$-axis is stretched on $0.5 \leq X / D \leq 3$ to show the slices more clearly. Compared to the wake of the helical-bladed turbine (figure 8), the streamwise vortical structures are symmetric about the $Z / D=0$ plane. Small counter-rotating secondary vortices are also present to the right of each main streamwise vortex, possibly similar to those observed in full-scale HAWTs by Yang et al. (2016).

the central updraft of fluid observed in figure 7(a). It will be argued in $\S 3.3$ that this asymmetry was a result of the blade twist of the helical-bladed turbine, which skewed the overall wake profile and thereby affected the alignment of the streamwise vortical structures.

Similar asymmetric behaviour in the wake of the helical-bladed turbine was observed in the vertical vortical structures $\left(\omega_{z}\right)$, shown in streamwise slices in figures 10 and 11 . In both cases, the structure with positively signed vorticity initially had a linear shape 

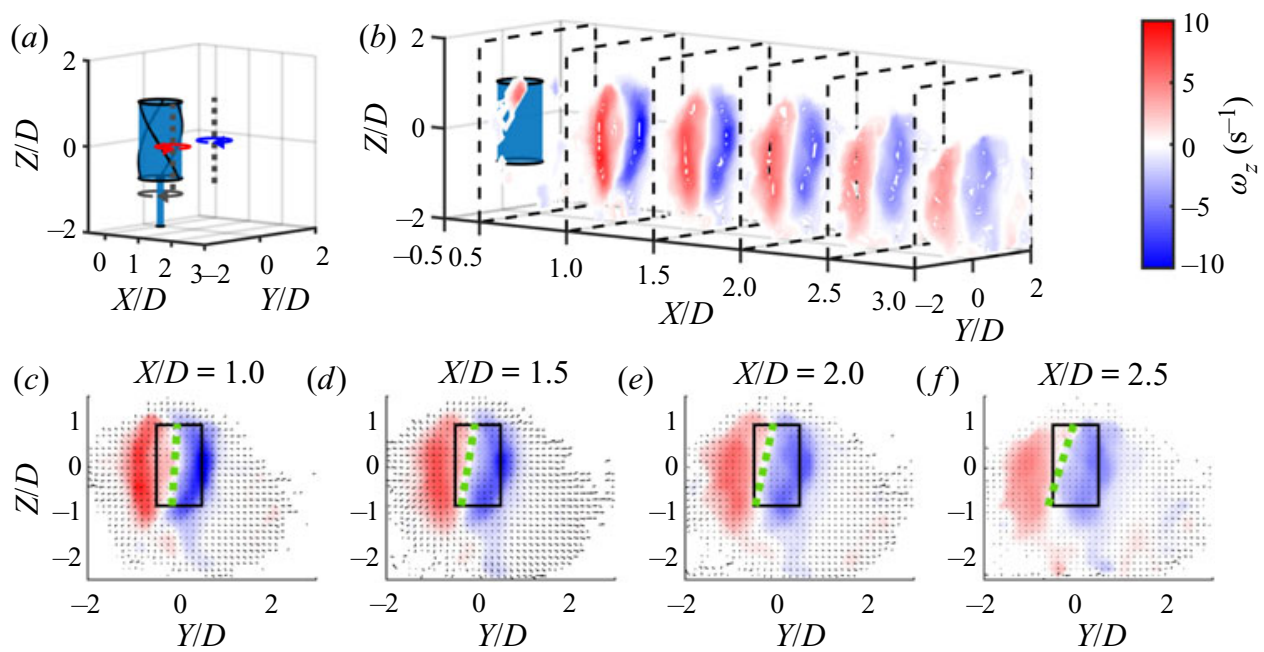

Figure 10. Streamwise slices of the vertical vorticity $\omega_{z}$ downstream of the helical-bladed turbine for $\lambda=1.19$. As in the previous figures, the $X$-axis is stretched on $0.5 \leq X / D \leq 3$. These structures exhibit a tendency to tilt with increasing streamwise distance from the turbine, as evidenced by fits to the zero-vorticity region between the structures (dashed green lines).

and was oriented vertically, while the structure with negatively signed vorticity was bent towards the positive $Y$ direction. This initial geometry was related to the mechanics of formation of these vortices. The positively signed structure was composed of vortices shed from turbine blades as they rotated into the wind, which formed a vortex line that was advected downstream. These vortices have been observed in two dimensions for straight-bladed turbines by Tescione et al. (2014), Parker \& Leftwich (2016) and Araya et al. (2017), and in 3-D simulations of straight-bladed turbines by Villeneuve et al. (2020). The arched shape of the negatively signed structures was a consequence of the low-velocity wake region. Behind the straight-bladed turbine, these two structures remained vertically oriented and relatively parallel. In contrast, behind the helical-bladed turbine, these structures began to tilt with respect to the vertical as they were advected downstream. This tilt was analogous to that observed in the horseshoe vortex (figure 8). Since the structures in $\omega_{z}$ were formed by vortex shedding from the turbine blades, we hypothesize that the helical blades of the UGE turbine were the cause of this observed asymmetric wake behaviour.

The spanwise vortical structures $\left(\omega_{y}\right)$ did not show any significant signs of asymmetry (appendix C.2, figures 28 and 29). These structures represented time-averaged tip vortices shed by the passing turbine blades, as documented by Tescione et al. (2014), and were thus not expected to change in geometry in these experiments. Together, the blade-shedding structures in $\omega_{y}$ and $\omega_{z}$ bounded the near wake. Their time-averaged profiles outlined and encapsulated the regions of streamwise-velocity deficit in the wake shown in figures 5 and 6. This topological correspondence suggested that vortex shedding from the turbine blades has a dominant effect on the overall shape of the near wake.

\subsection{Effect of blade twist}

In the previous section, connections between vortex shedding from VAWT blades and the 3-D topology of VAWT wakes were observed. A more thorough investigation of the tilted wake is now undertaken to develop a more comprehensive description of the dynamics in the near wake. 
(a)

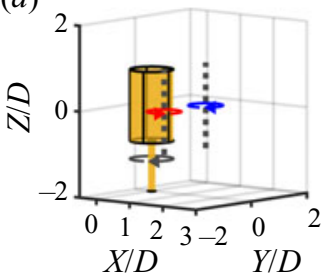

(b)

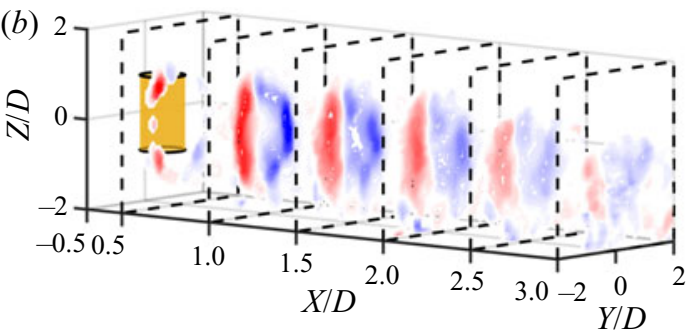

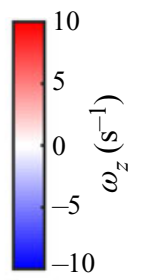

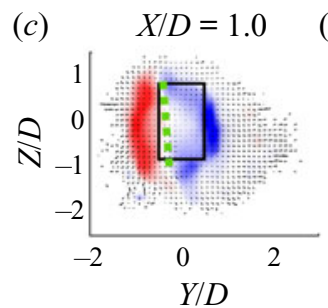

(d)
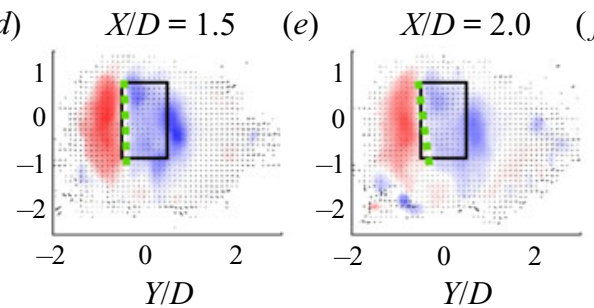

$(f)$

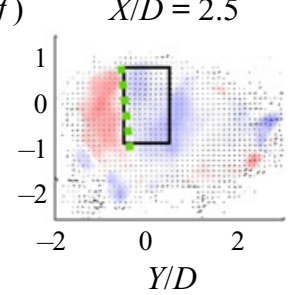

Figure 11. Streamwise slices of the vertical vorticity $\omega_{z}$ downstream of the straight-bladed turbine for $\lambda=1.20$. The $X$-axis is again stretched on $0.5 \leq X / D \leq 3$. These structures remain upright with respect to the vertical (again denoted by dashed green lines), in contrast to their counterparts from the helical-bladed turbine.

The results presented thus far have shown that the wake of the helical-bladed turbine was tilted at some angle with respect to the vertical, whereas the wake of the straight-bladed turbine was not. To quantify this effect, two measures were employed: the angle of the velocity-deficit region, and the angle of the region of zero vorticity between the two vertical vortical structures. These two measures were selected on the premise that the dynamics of the vertical vortical structures is tied to the geometry of the near wake. Both were computed on slices parallel to the $Y Z$ plane, taken at several streamwise positions downstream of each turbine. For the first measure, the location of minimum velocity was identified at every Z-position in each slice, and a linear fit through these points on each slice was computed to approximate the slope of the velocity-deficit region. For the second measure, the location of minimum vorticity between the two vertical vortical structures was identified at every Z-position in each slice using linear interpolation, and a linear fit through these points on each slice represented the orientation of the structures. For both measures, confidence intervals of one standard deviation on the slope of the linear fit served as error bounds. The results of this procedure are shown in figure 12(a) for the velocity-deficit measure and figure $12(b)$ for the vortical-structure measure. The results demonstrated that the wake orientation of the straight-bladed turbine did not exhibit a strong deviation from the vertical in the near-wake region. In contrast, the wake orientation of the helical-bladed turbine increased monotonically with streamwise distance for both of the tip-speed ratios tested in the experiments. These measures thus quantified the various observations from the previous section regarding changes in the wake topology between the two turbines.

The wake-orientation measurements shed light on the mechanism responsible for the tilted wake observed for the helical-bladed turbine. Firstly, the orientation profiles were very consistent between the two measures, supporting the hypothesis that the shape of the near wake is directly tied to the dynamics of the vertical vortical structures. Additionally, the profiles did not show a strong dependence on turbine solidity, and no significant dependence on tip-speed ratio over the limited range of $\lambda$ tested in these experiments was observed. Though the measurements did separate into two classes corresponding to the 


\section{N.J. Wei and others}

(a)

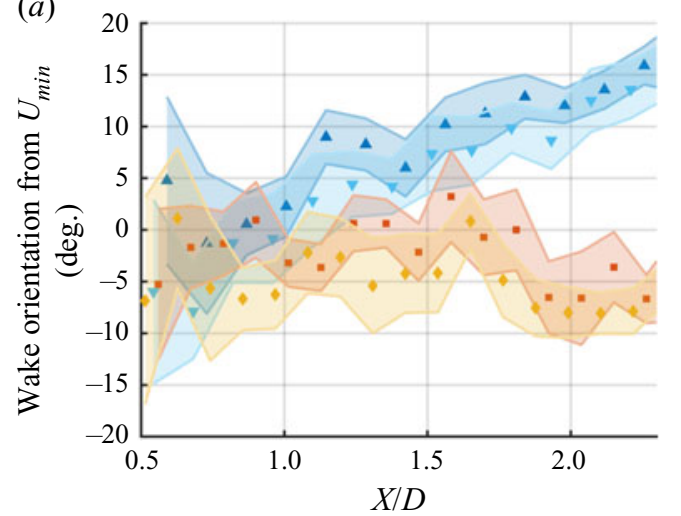

(b)

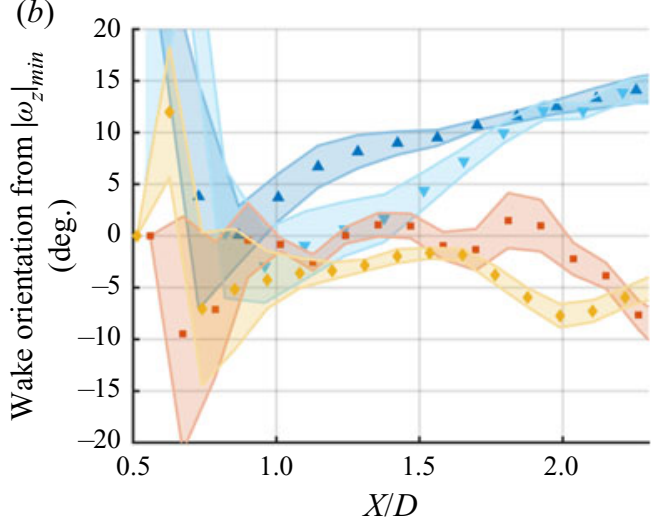

$\triangle$ UGE, $\lambda=1.19 \quad$ "WPE, $\lambda=0.96$

$\checkmark \mathrm{UGE}, \lambda=1.40 * \mathrm{WPE}, \lambda=1.20$

Figure 12. Wake-orientation measurements from all four experimental cases, computed from $(a)$ the locations of minima in the velocity-deficit region, $U_{\min }$, and $(b)$ the coordinates of the zero-vorticity strip between the two vertical vortical structures, $\left|\omega_{z}\right|_{\text {min }}$. The wake orientation of the helical-bladed turbine increases monotonically, while that of the straight-bladed turbine does not exhibit a strong trend away from zero.

two turbine geometries, the three-dimensional nature of the tilted-wake behaviour made it unlikely that solidity, which is not defined using three-dimensional geometric parameters, was the dominant factor. These results thus isolate the geometry of the turbine blades as the primary contributing factor to the observed differences in wake topology.

A mechanism by which turbine-blade geometry can affect the wake topology is now proposed. As described previously, the vortical structures visible in the time-averaged fields of $\omega_{z}$ represent the profiles of vortex lines shed from the rotating turbine blades and advected away from the turbine by the free stream. We expect that the shape of these vortex lines will depend on the shape of the blades: a straight blade will shed a straight vortex line, while a helical blade will shed a vortex line with non-zero curvature. The latter hypothesis is drawn from the fact that a helical blade, in contrast to a straight blade, is yawed relative to the incoming flow and experiences a range of local angles of attack along its span as it rotates around the turbine. A yawed or swept blade with respect to the incoming flow exhibits strongly three-dimensional vortex shedding in dynamic stall (Visbal \& Garmann 2019), due in part to a net transport of vorticity along the span of the blade (Smith \& Jones 2019). A linearly increasing spanwise angle-of-attack profile in dynamic stall similarly induces a spanwise transport of vorticity that affects the stability of the leading-edge vortex and thus the character of the vortex shedding from the blade (Wong, laBastide \& Rival 2017). Given these observations, we conclude that the spanwise non-uniformity of the flow over helical VAWT blades makes the vortex lines shed by the dynamic-stall mechanism inherently nonlinear and three-dimensional.

To model the evolution of these vortex lines as they are advected downstream, the principle of Biot-Savart self-induction can be applied. In an inviscid flow field, the self-induced velocity at any point $\boldsymbol{r}$ on a single vortex line with finite core size $\mu$, defined by the curve $\boldsymbol{r}^{\prime}$ and parameterized by the arc length $s^{\prime}$, can be written as

$$
\frac{\partial \boldsymbol{r}}{\partial t}=-\frac{\Gamma}{4 \pi} \int \frac{\left(\boldsymbol{r}-\boldsymbol{r}^{\prime}\right) \times \frac{\partial \boldsymbol{r}^{\prime}}{\partial s^{\prime}}}{\left(\left|\boldsymbol{r}-\boldsymbol{r}^{\prime}\right|^{2}+\mu^{2}\right)^{3 / 2}} \mathrm{~d} s^{\prime},
$$


where the integration is performed over the length of the vortex line (Leonard 1985). This model for the self-induced deformation of curved vortex lines has been studied numerically using both approximate methods (Arms \& Hama 1965) and exact simulations (Moin, Leonard \& Kim 1986). The quantity $\left(\boldsymbol{r}-\boldsymbol{r}^{\prime}\right) \times \partial \boldsymbol{r}^{\prime} / \partial s^{\prime}$ in the numerator of the integrand is only non-zero when the displacement vector between two points on the vortex line does not align with the direction of the vortex line. Therefore, a vortex line with curvature or piecewise changes in alignment will undergo deformation under self-induction, while a purely linear vortex line will not. Self-induced deformations of a similar nature have been observed in curved and tilted vortex lines in numerous computational and experimental contexts (e.g. Hama \& Nutant 1961; Boulanger, Meunier \& Dizès 2008).

Given that the precise shape of the curved vortex lines shed by the helical-bladed turbine cannot be extracted from the time-averaged vorticity fields, (3.1) cannot be applied quantitatively in this case. However, it can still be used qualitatively to connect the development of the tilted wake to blade geometry. We therefore consider a helical vortex line that corresponds to the helical shape of the UGE turbine blades, shed from a blade as it rotates upstream into the prevailing wind. This model system accounts for the three-dimensional blade geometry while abstracting the precise dynamics of the vortex-shedding mechanism on the blades. The curve is parameterized for $Z \in$ $[-S / 2, S / 2]$ as $X=-a R \sin (\tau Z)$ and $Y=-R \cos (\tau Z)$. The constant $a$ accounts for stretching of the vortex line in the streamwise direction due to differences between the tip-speed velocity of the turbine and advection from the free stream. The selection $a=1 / \lambda$, for example, recovers the expected asymptotic result that the curved vortex line will become a straight vertical line as $\lambda \rightarrow \infty$. The initial induced velocities along this vortex line, computed numerically from (3.1), apply a stretching in the $Y$ direction that corresponds directly with the previous observations of the tilted wake (figure 13). The streamwise and vertical induced velocities are not addressed in this analysis since they do not contribute to the tilted wake. The computed vectors also do not represent the full time evolution of the vortex line, as only the initial induced velocities $\boldsymbol{u}(\boldsymbol{r}(t=0))$ are given. The demonstration shows qualitatively that the mechanism of Biot-Savart self-induction provides a direct connection between blade geometry and the evolution of the wake topology.

Based on these results, the propagation of the influence of turbine-blade geometry in the dynamics of the near wake can be outlined. The shape of the blades determines the shape of the vortex lines shed by the blades as they rotate around the turbine. The shape of these vortex lines in turn drives their evolution downstream of the turbine. As these structures bound the wake region, topological changes in the vortex lines are reflected in the shape of the velocity-deficit region of the wake. These developments affect the shape of the horseshoe vortex, which is not directly affected by differences in blade geometry but is necessarily bound to the shape of the wake region. The connection between blade twist and wake tilt described in this section therefore provides a unifying framework that accounts for the trends previously observed in the wake velocity and vorticity fields.

\subsection{Wake dynamics}

In the previous section, it was hypothesized that the shape of VAWT blades dictates the near-wake topology through Biot-Savart self-induction of shed vortex lines. The wake dynamics is now analysed in detail, to quantify the evolution of the vortical structures in the wake and to identify their contributions to wake recovery. The circulation of the wake structures in each direction, $\Gamma_{i}$, was calculated as a function of downstream distance from the turbine. The circulation was computed at a series of streamwise 


\section{N.J. Wei and others}

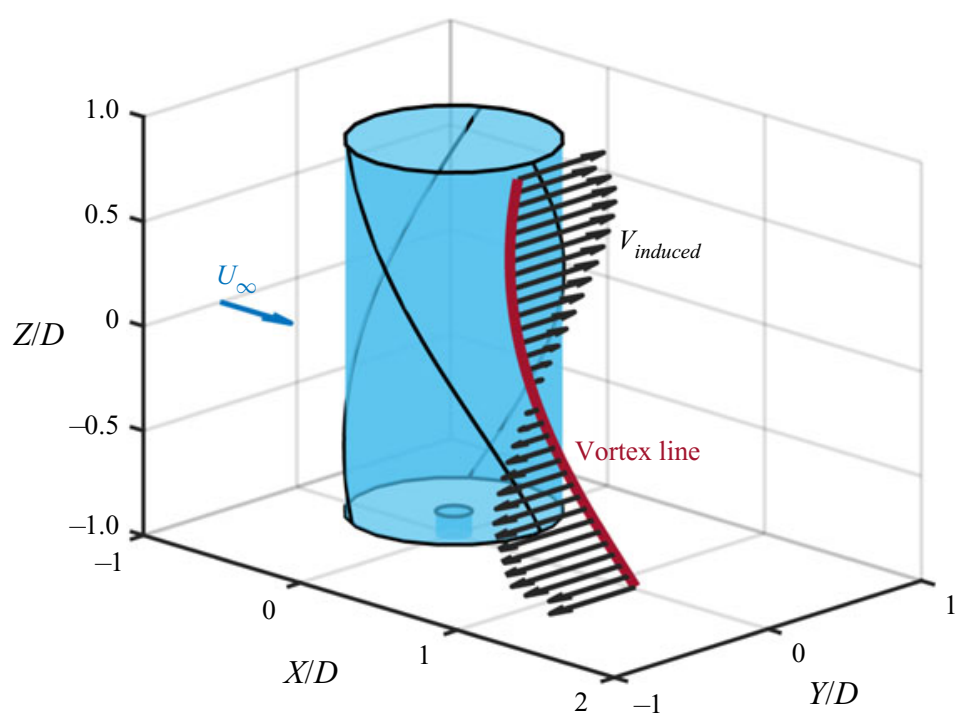

Figure 13. Schematic of the $Y$ component of the induced velocities, $V_{\text {induced }}$, along a helical vortex line due to Biot-Savart self-induction (3.1). The scale of the vectors and the streamwise location of the vortex line are both arbitrary, and the streamwise and vertical components of the induced velocity are not shown for clarity. The stretching induced on the vortex line matches the behaviour of the tilted wake.

positions $X / D$ by integrating the vorticity component in question over a square $D \times D$ window, which was oriented normal to the direction of the vorticity component. This window was placed at the centre of the vortex at several points along the vortex line. The centre was identified at each point by applying a threshold based on the standard deviation of the vorticity field and computing the centre of mass of the isolated vorticity distribution. The values of the circulation along the vortex line, computed with these windows, were averaged to obtain a representative circulation for the structure. Error bars were determined by propagating the standard deviations of the components of individual velocity vectors from the particle trajectories in each voxel through the curl operator and the circulation integration. This computation was done independently for the positively and negatively signed vortical structures. The resulting circulations in $X, Y$ and $Z$ are plotted on $0.5 \leq X / D \leq 2.3$ in figures $14,15(a)$ and $15(b)$, respectively. Circulation measurements downstream of this region were inconclusive due to measurement noise, or possibly as a result of atmosphere-induced unsteady modulations in the wake similar to those observed in full-scale HAWTs (Abraham \& Hong 2020).

The circulations of the streamwise structures $\left(\Gamma_{x}\right.$, figure 14) appeared to extend farther into the wake than the vortical structures in $Y$ and $Z$, whose circulations declined monotonically after $X / D \approx 1.5$ (as shown in figure 15 ). This difference in dynamical behaviour agrees with the previously stated hypothesis that the horseshoe vortex is induced by the rotation of the turbine rather than unsteady vortex shedding from the turbine blades. Furthermore, the streamwise circulation at the lowest tip-speed ratio $(\lambda=0.96)$ began to decline at $X / D \approx 1.7$, prior to those at higher tip-speed ratios, which suggests that the persistence of the horseshoe vortex in the wake increases with increasing $\lambda$. Given the limited range of tip-speed ratios and streamwise locations considered in these experiments, a definitive relation between $\Gamma_{x}$ and $\lambda$ cannot be isolated from these results. Still, the comparatively long-lived coherence of the horseshoe vortex in the wake does imply that it plays an important role in the dynamics of wake recovery, considering its influence 


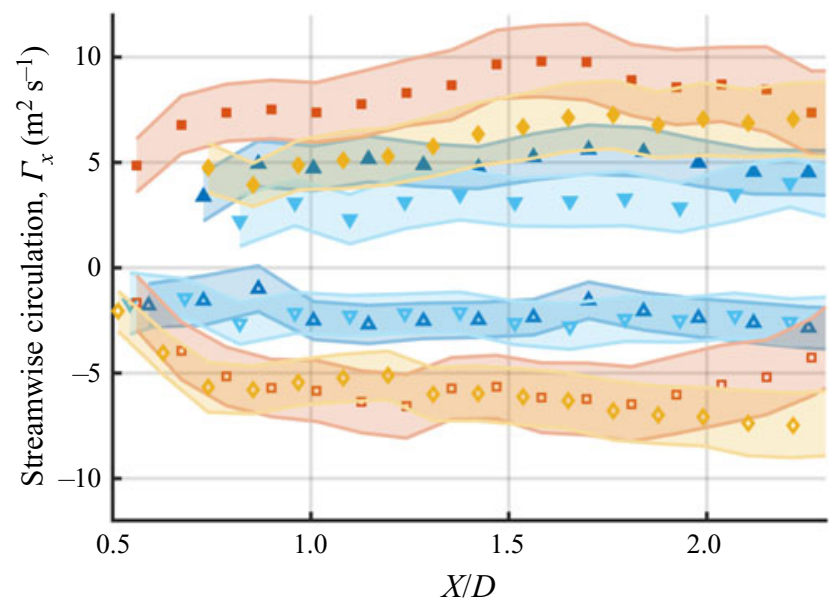

\begin{tabular}{|ll|}
\hline$\Delta$ UGE, $\lambda=1.19(+)$ & $\because$ WPE, $\lambda=0.96(+)$ \\
$\Delta$ UGE, $\lambda=1.19(-)$ & WPE, $\lambda=0.96(-)$ \\
$\nabla$ UGE, $\lambda=1.40(+)$ & $\diamond$ WPE, $\lambda=1.20(+)$ \\
$\nabla$ UGE, $\lambda=1.40(-)$ & $\diamond$ WPE, $\lambda=1.20(-)$
\end{tabular}

Figure 14. Circulation of the positive and negative streamwise vortical structures, $\Gamma_{x}$, for all four experimental cases. Compared to the plots of $\Gamma_{y}$ and $\Gamma_{z}$ shown in figure 15, $\Gamma_{x}$ did not decay as significantly with increasing streamwise distance, and the horseshoe vortex was thus hypothesized to extend farther into the wake than the structures induced by vortex shedding from the blades.

on vertical velocities in the wake and thus the entrainment of momentum into the wake (figure 7). Additionally, the difference in the alignment of the branches of the horseshoe vortex between the helical- and straight-bladed turbines suggests that the wake recovery will also differ as a function of blade geometry.

The circulations of the structures corresponding to vortex shedding from the turbine blades ( $\Gamma_{y}$ and $\Gamma_{z}$, shown in figures $15 a$ and $15 b$ ) exhibited uniform behaviour for both turbines. Taken together with the time-resolved results of Araya et al. (2017), these trends confirm that the vortex-shedding dynamics that contributes to the wake structure is only active in shaping the near wake $(X / D \lesssim 2)$. An apparent dependence on turbine solidity is evident in these data, as the circulation profiles separated into groups by turbine rather than by tip-speed ratio. This trend is likely not a function of three-dimensional blade shape, because the measurements of $\Gamma_{y}$ and $\Gamma_{z}$ were averaged in $Y$ and $Z$, respectively. Again, because of the limited number of turbine geometries considered in this study, a definitive relation between $\Gamma_{y}, \Gamma_{z}$ and $\sigma$ cannot be established from these data. These results, however, do indicate that the contributions of the blade-shedding structures to wake recovery will not differ significantly between the two turbine geometries considered in this study.

The main conclusion from the circulation analysis presented in this section is that the horseshoe vortex extends farther into the wake than the vortical structures in the $Y$ and $Z$ directions, which begin to decay in strength soon after they are shed from the turbine blades. These results thus suggest that, though the vortex lines shed by the turbine blades define the topology of the near wake, the horseshoe vortex has a larger contribution to wake recovery due to its comparative longevity in the wake. The analysis also noted possible dependencies of $\Gamma_{x}$ on $\lambda$ and of $\Gamma_{y}$ and $\Gamma_{z}$ on $\sigma$. The contributions of vortex breakdown and turbulent entrainment of momentum from the free stream to wake recovery 


\section{N.J. Wei and others}

(a)

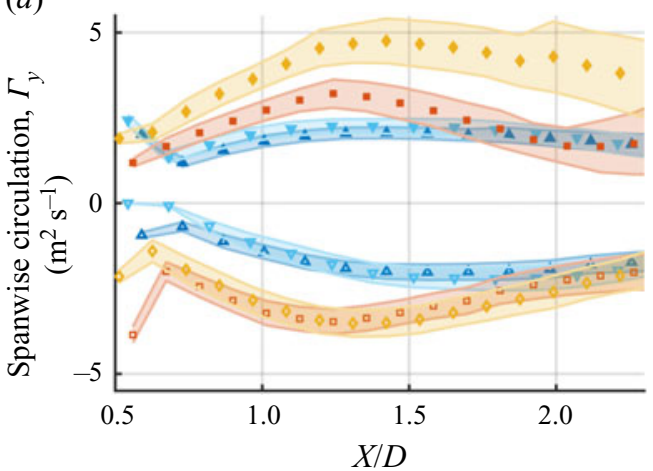

(b)

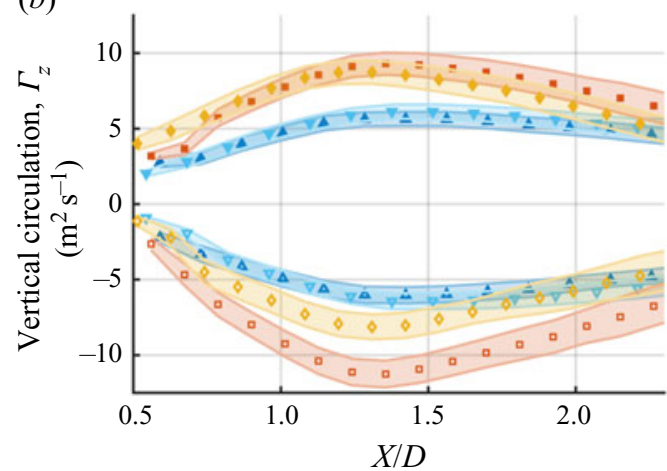

$\triangle \mathrm{UGE}, \lambda=1.19(+) \quad$ WPE, $\lambda=0.96(+)$
$\triangle \mathrm{UGE}, \lambda=1.19(-) \quad$ WPE, $\lambda=0.96(-)$
$\nabla \mathrm{UGE}, \lambda=1.40(+) \quad *$ WPE, $\lambda=1.20(+)$
$\nabla \mathrm{UGE}, \lambda=1.40(-) \quad \circ$ WPE, $\lambda=1.20(-)$

Figure 15. Circulations of the positive and negative $(a)$ spanwise vortical structures $\left(\Gamma_{y}\right)$ and $(b)$ vertical vortical structures $\left(\Gamma_{z}\right)$ for all four experimental cases. The circulation profiles collapse approximately by turbine, implying that turbine solidity is a dominant factor in this dynamics. The decaying trends of the profiles past $X / D \approx 1.5$ show that these structures are only influential in the near wake.

have not been considered here, because of the time-averaged nature of the experimental data. These effects become more significant downstream of the limit of our measurement domain, and thus will also be affected by the mean-flow dynamics identified in this work.

\subsection{Implications for turbine arrays}

The findings of this study have parallels in recent studies of the wake dynamics of HAWTs that highlight the implications of the present results for the arrangement of VAWTs in closely packed arrays. In both experiments with a porous disc and large-eddy simulations with actuator-disc and actuator-line models, Howland et al. (2016) reported an alteration to the shape of the wake profile, known as a curled wake, behind HAWTs yawed with respect to the incoming flow caused by counter-rotating streamwise vortices that are induced by the yawed turbine. Bastankhah \& Porté-Agel (2016) and Shapiro, Gayme \& Meneveau (2018) modelled this topological phenomenon analytically, leading to new estimates of the wake deflection and available power downwind of a yaw misalignment wind turbine. Fleming et al. (2017) confirmed the existence of these vortices for yawed turbines in field conditions. Additionally, Fleming et al. (2018) demonstrated that these changes in wake topology affect the performance of downstream and adjacent turbines in an array. Howland, Lele \& Dabiri (2019) and Fleming (2019) then utilized these analytic models to demonstrate the potential for wake steering control in field experiments of HAWTs at full scale. Given the qualitative similarities in the dynamics of the tilted wake observed in this study and the curled wake observed in HAWTs, the ramifications of the tilted wake for arrays of VAWTs could be analogous as well.

The performance of VAWTs downstream of a given turbine will be affected by the dynamics identified in this study. The strong vortex shedding in the near wake of a VAWT $(X / D \lesssim 2)$ will lead to a severe decrease in the performance of downstream turbines placed in this region. Brownstein et al. (2019) observed this effect in experiments with turbine pairs. For greater streamwise separations, the horseshoe vortex will be the primary 
large-scale mean-flow vortical structure encountered by downstream turbines. At these distances, the effects of helical blades on wake recovery will be more evident, and will affect the overall performance of the VAWT array. The tilted wake will also lead to a modified wake profile that will affect the optimal placement of helical-bladed turbines in arrays by at least one turbine radius, in a manner similar to that reported by Bossuyt et al. (2016) for HAWTs.

The performance of adjacent VAWTs will also be affected, especially for turbines placed in close proximity to each other for the enhancement of power production. The mean-flow mechanisms observed by Brownstein et al. (2019) will be altered by the presence of the three-dimensional vortex shedding from helical-bladed turbines, and it is thus likely that these turbines would experience a different degree of performance enhancement observed in that work for straight-bladed turbines.

Lastly, the vortical structures and topological effects isolated in this study are expected to hold for vertical-axis wind turbines in general, with variations for different turbine geometries. The horseshoe vortex, in particular, is expected to be enhanced for VAWTs that operate at higher tip-speed ratios than those employed in these experiments. The significance of the tilted wake for helical-bladed turbines will vary with aspect ratio and blade twist. The 3-D effects of blade geometry will also need to be reconsidered for the bowed blades of the large-scale Darrieus-type turbines studied by Klimas \& Worstell (1981). Particular differences notwithstanding, it can be inferred that the three-dimensional dynamics identified here will have significant effects on the dynamics and topology of the wake for VAWTs of all designs and power outputs, and thereby affect the aerodynamics and overall efficiency of VAWT arrays.

\section{Conclusions}

In this study, a novel method for conducting volumetric three-dimensional, three-component flow-field measurements in field conditions was developed and used to quantify the wake dynamics of two full-scale vertical-axis wind turbines. This 3-D PTV set-up demonstrated sufficient precision and resolution to resolve large-scale vortical structures in the wakes of the turbines. Time-averaged velocity and vorticity fields showed a tilted wake downstream of helical-bladed turbines, compared to the wake profile of straight-bladed turbines. A fully three-dimensional analysis of the vortical structures present in the near wakes of VAWTs was undertaken, and demonstrated that the topology of the near wake is dependent primarily on the mechanics of vortex shedding from the turbine blades. The connection between blade geometry and wake dynamics was clarified by considering the Biot-Savart self-induction of curved vortex lines. Measurements of the circulation of the vortical structures in the wake revealed that the vortical structures shed by the blades decay in influence relatively quickly but affect the topology of the horseshoe vortex, which extends farther into the wake and is thus related to wake recovery. Therefore, a line of influence was established between blade geometry, vortex shedding, near-wake topology, the horseshoe vortex and wake recovery.

A major point of emphasis in this work is that three-dimensional effects are significant to the dynamics and evolution of VAWT wakes. Many of the flow phenomena observed in this study would be difficult to resolve completely by planar measurement techniques; the vorticity and circulation measurements in particular required three-component 3-D data to collect. The experimental method for obtaining time-averaged velocity and vorticity measurements in field conditions and high Reynolds numbers is therefore well suited to further analyses of wind-turbine wake dynamics at full scale. The method is also 


\section{N.J. Wei and others}

flexible enough in its deployment to be applied to other large-scale flow phenomena and atmosphere-structure interactions.

While this study focused on differences in flow topology that stemmed from the use of helical blades in VAWTs, the connection between object geometry and vortex dynamics proposed in this work could be applied in wider aerodynamic contexts, such as rotorcraft, flapping flight and more complex fluid-structure interaction problems. This study provides both an experimental method and a theoretical framework for wake analysis that can be leveraged for future studies of the three-dimensional vortex dynamics in engineering-scale wakes.

The limited number of test cases in this study precluded the execution of a full scaling analysis of the vortical structures and circulation profiles with respect to turbine solidity and tip-speed ratio. A larger series of test cases, involving different turbine geometries and a wider range of tip-speed ratios, would allow these relationships to be established more quantitatively. In addition, a larger measurement volume would allow the wake-topology observations of this study to be extended into the far wake, so that the effects of the tilted wake and the streamwise vortical structures on wake recovery could be more thoroughly investigated. Given the size of the measurement domain that would be required for these kinds of experiments, as well as the range of parameters involved, it may be more feasible to carry out this analysis in a laboratory or computational setting. This study can thus provide validation cases to demonstrate that flow phenomena observed in VAWT wakes in laboratory experiments and numerical simulations are representative of those present in the wake of operational turbines in field conditions.

Acknowledgements. The authors gratefully acknowledge funding from the Gordon and Betty Moore Foundation through grant no. 2645, the National Science Foundation through grant no. FD-1802476, the Stanford University TomKat Center for Energy Sustainability and the Stanford Graduate Fellowships in Science and Engineering. The authors would also like to recognize B. Hayes and Prevailing Wind Power for managing the operation and maintenance of the FLOWE field site, and R. McMullen for assisting with the field experiments. The authors extend their thanks to Snow Business International \& Snow Business Hollywood for assisting in the selection and supply of the snow machines and the snow fluid used, for providing troubleshooting during late-night experiments, for allowing extended use of their machines and for shipping a machine to Stanford for the laboratory experiments. Lastly, the authors would like to extend their appreciation to the reviewers from the Journal of Fluid Mechanics for their detailed feedback and helpful suggestions that significantly improved the presentation of this work.

Declaration of interests. The authors report no conflict of interest.

Author ORCIDs.

(1) Nathaniel J. Wei https://orcid.org/0000-0001-5846-6485;

(ㄷ) Michael F. Howland https://orcid.org/0000-0002-2878-3874.

\section{Appendix A. Dynamics of artificial snow particles}

Since the choice of flow seeding particles is critical to the accuracy of PTV measurements, the generation and characterization of the artificial snow particles used in these experiments were carefully considered. Natural snowfall had been used successfully by Hong et al. (2014) as seeding particles for 2-D particle-image velocimetry in the wake of a full-scale HAWT. In the present experiments, artificial snow was used due to the lack of natural snowfall at the FLOWE site. The artificial snow used in these experiments was composed of an air-filled soap foam (ProFlake Falling Snow Fluid, Snow Business), which formed irregularly shaped particles with a range of sizes. The particles that were visible in the images from the field site had diameters of $d_{p}=11.2 \pm 4.2 \mathrm{~mm}$ and an average density of $\rho=6.57 \pm 0.32 \mathrm{~kg} \mathrm{~m}^{-3}$. Smaller particles would have been more 
ideal as 'tracer' particles, but these were difficult to visually identify and isolate in the recorded videos and were thus unfeasible for these experiments. The settling velocity of these particles in quiescent air was measured to be $W_{s}=0.60 \pm 0.18 \mathrm{~ms}^{-1}$, which corresponds to a particle Reynolds number $R e_{p}=W_{s} d_{p} / v$ of 448 . Since this Reynolds number is outside the Stokes-flow regime, the relative influence of inertial effects on the ability of the particles to follow the flow had to be ascertained. A considerable body of research documents the dynamics of various types of inertial particles in turbulence (e.g. Böhm 1988; Aliseda et al. 2002; Xu \& Bodenschatz 2008; Toschi \& Bodenschatz 2009; Bourgoin \& Xu 2014; Ireland, Bragg \& Collins 2016; Nemes et al. 2017; Petersen, Baker \& Coletti 2019). Regardless, the foam-based composition and irregular shapes of the artificial snow particles meant that laboratory experiments were required to more accurately characterize their dynamics.

In this section, a series of laboratory experiments are detailed that allowed the flow response of the artificial snow particles to be quantified. This is followed by a qualitative analysis based on scaling arguments that confirms the findings of the laboratory experiments in the field data.

\section{A.1. Laboratory experiments with a snow machine}

Laboratory experiments in a wind tunnel were designed to determine the aerodynamic response characteristics of the artificial snow particles used in the field experiments. To measure the particle response to a step change in velocity, a snow machine was arranged to release particles normal to the flow in the wind tunnel, creating a jet in cross-flow. As this canonical flow has been studied extensively in experiments, simulations and analytical investigations (cf. Fric \& Roshko 1994; Gopalan, Abraham \& Katz 2004; Su \& Mungal 2004; Mahesh 2013; Karagozian 2014), it served as a well-documented baseline against which the particle dynamics could be evaluated. The spanwise discrepancy between the profile of the jet in cross-flow and the particle trajectories could then be analysed to establish a particle relaxation time and quantify the effects of particle inertia on flow fidelity. The dynamics of the large particles used in the field experiments was therefore compared with the behaviour of smaller, more regularly shaped particles generated by increasing the blower flow rate and decreasing the fluid injection rate in the snow machine. The response of each particle type was studied at two distinct free-stream velocities. The impulse response and slip velocity of the particles were then calculated from the measured jet profiles.

Experiments to characterize the dynamics of particles produced by a single snow machine (Silent Storm DMX, Ultratec Special Effects) were conducted in an open-circuit wind tunnel with flow driven by a $4 \times 4$ grid of fans at the inlet of the tunnel. The test section was $4.88 \mathrm{~m}$ long in the streamwise direction $(X), 2.06 \mathrm{~m}$ in width $(Y)$ and $1.97 \mathrm{~m}$ in height $(Z)$. The tunnel was operated with free-stream velocities of $5.64 \pm 0.45$ and $6.58 \pm 0.45 \mathrm{~ms}^{-1}$, as measured with a hot-film anemometer. A full description of the wind-tunnel facility is given by Brownstein et al. (2019).

The snow machine was positioned on a platform $2.8 \mathrm{~m}$ downstream of the tunnel inlet, so that the output nozzle was perpendicular to the centreline of the tunnel (cf. figure 16). The end of the nozzle was offset at $Y=-0.2 \mathrm{~m}$ in the spanwise direction from the centre of the tunnel. The blockage from this configuration was less than $5 \%$. Four hardware-synchronized cameras $(\mathrm{N}-5 \mathrm{~A} 100$, Adimec) were positioned around the tunnel, with one located directly above the measurement volume. These cameras captured a measurement volume of approximately $1.2 \mathrm{~m} \times 0.5 \mathrm{~m} \times 0.5 \mathrm{~m}$ in the $X, Y$ and $Z$ 


\section{N.J. Wei and others}

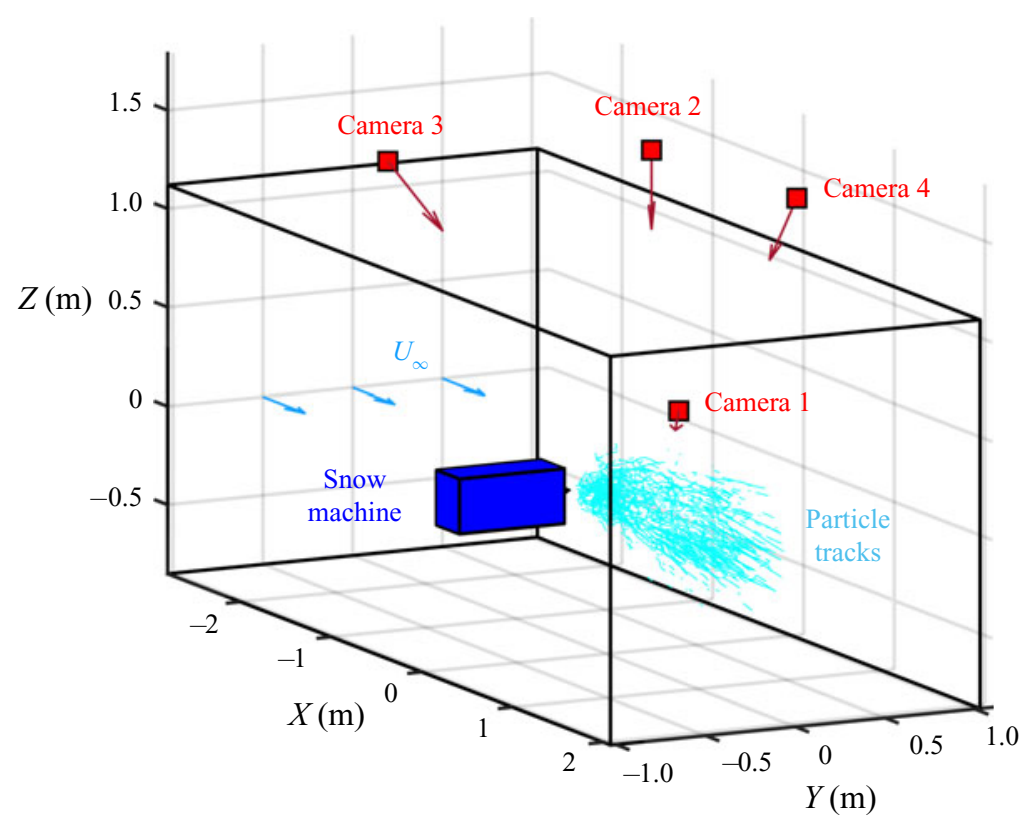

Figure 16. Schematic of the experimental set-up for snow-machine experiments. Flow is in the positive $X$ direction, and the snow machine (blue) emits particles in the positive $Y$ direction. Sample particle tracks (light blue) illustrate the extent of the measurement volume. The four cameras are shown in red, and arrows denote their viewing angles.

directions, respectively. The cameras recorded images at a resolution of $2048 \times 1008$ pixels and a frequency of 250 frames per second, with an exposure of $600 \mu \mathrm{s}$.

Three-dimensional PTV was used to quantify the movements of the snow particles. A wand-based calibration procedure, identical to that used in the field experiments (given in appendix B.1), was used to reconstruct particle tracks in three dimensions. A wand composed of a pair of LEDs spaced $10 \mathrm{~cm}$ apart was moved throughout the measurement volume, and the calibration utility of Theriault et al. (2014) established the camera positions from image coordinates of the two lights in each camera view. The wand length was reconstructed with an error of $0.75 \%$, suggesting that the calibration was sufficiently accurate. The global coordinate system was set using a plumb line at the centre of the wind tunnel, so that the nozzle was located at $(X, Y, Z)=(0,-0.2,0) \mathrm{m}$. Particles were identified, triangulated in physical space, and tracked using the procedure outlined in appendix B.2. Because of the high seeding density of the particles, only the largest $10 \%$ by area of the identified particles in the raw images were triangulated into physical coordinates. This was done to reduce triangulation ambiguities, and to isolate the large particles that would have been observable in the field experiments for analysis. A statistical analysis of the size distributions of the particles from the two output settings was achieved by binarizing images taken by the camera positioned directly above the measurement volume (figure 17), and converting the pixel areas of the identified particles to effective diameters in physical dimensions. The largest $10 \%$ of the particles by area had effective diameters of $d_{p}=11.2 \pm 4.2 \mathrm{~mm}$ in the large-snow case and $d_{p}=5.9 \pm 1.1 \mathrm{~mm}$ in the small-snow case. For each experiment, at least 120 seconds of data were recorded, corresponding to over 30000 raw images per camera. The resulting velocity vectors were binned and time-averaged into 3 -cm cubic voxels with at least 10 vectors per displayed voxel. 


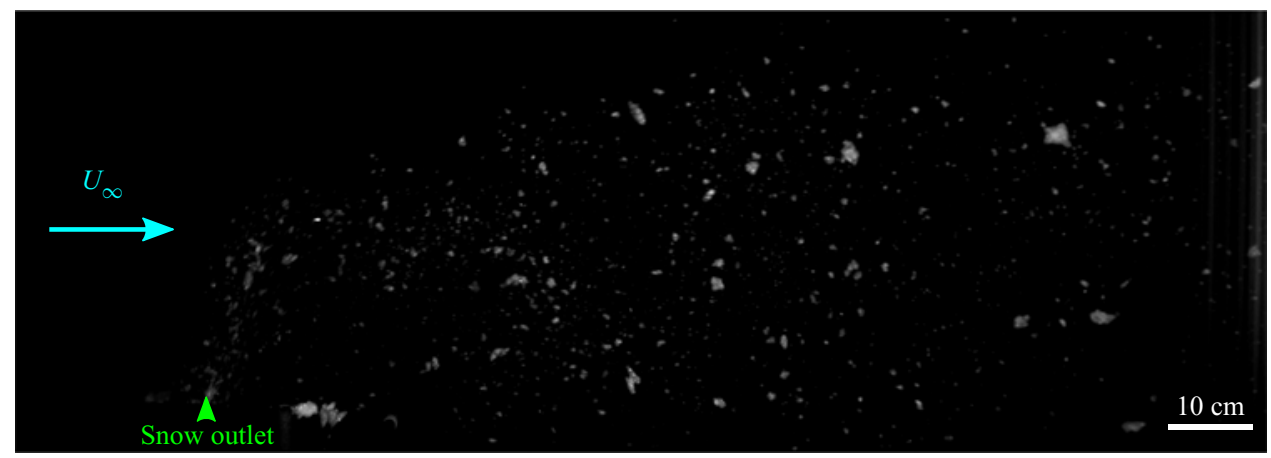

Figure 17. Photograph of snow particles of the type used in the field experiments, viewed from above (Camera 2). Particles are generated at the nozzle at the lower left (green arrow) and are convected toward the right of the image.

\section{A.2. Experimental results}

Particle time scales and response characteristics were computed from the data in the following manner. Firstly, the jet in cross-flow was identified from velocity fields measured with the small snow particles. This was accomplished by applying a high-pass filter to streamwise slices of the spanwise velocity $V$ to isolate the jet in cross-flow from the signal of the larger particle jet. A hill-climbing search algorithm was then applied within this region to find the local maximum of spanwise velocity corresponding to the centre of the jet at each streamwise location. These maxima were projected into the $X Y$ plane to define the jet centreline (figure 18). To educe a jet profile from these data, it was assumed that the trajectory of the jet in cross-flow would follow the similarity solution given by Hasselbrink \& Mungal (2001) for the region near the jet orifice

$$
\frac{x}{r d}=\left(\frac{2}{c_{e j}} \frac{y}{r d}\right)^{1 / 2} .
$$

The jet diameter $d$ was estimated as $3 \mathrm{~cm}$ based on the diameter of the snow-machine particle generator, and the velocity ratio $r=V_{\text {jet }} / U_{\infty}$ was calculated based on the maximum velocity measured on the jet centreline. A single-parameter fit for the measured jet centreline was then applied to estimate the entrainment coefficient, $c_{e j}$. Between the two small-snow experiments $(r=0.29$ and 0.36$)$, the extracted entrainment coefficient was $c_{e j}=0.39 \pm 0.01$. This was slightly larger than the value of $c_{e j}=0.32$ given by Ricou \& Spalding (1961) for a free jet in controlled conditions, likely due to the higher turbulence intensities in this experiment. The jet in cross-flow in the large-snow experiments was harder to identify in the velocity fields because of its lower velocity ratio (a consequence of the lower snow-machine blower setting), which resulted in a lower signal-to-noise ratio. The presence of instabilities in the jet at low velocity ratios likely further obscured the jet profile in the time-averaged velocity fields (Ilak et al. 2012; Klotz, Gumowski \& Wesfreid 2019). Thus, for the sake of consistency across experiments, the measured value for $c_{e j}$ from the small-snow experiments was used to infer the trajectory of the jet in cross-flow in the large-snow experiments.

The particle jet was identified using images taken from above the measurement domain (figure 19). The particles detected in each frame were binned into 3-cm square areas and were plotted as a 2-D distribution of particles, averaged over all images in the experiment. The centre of the jet was identified at each streamwise location to subpixel accuracy using 


\section{N.J. Wei and others}

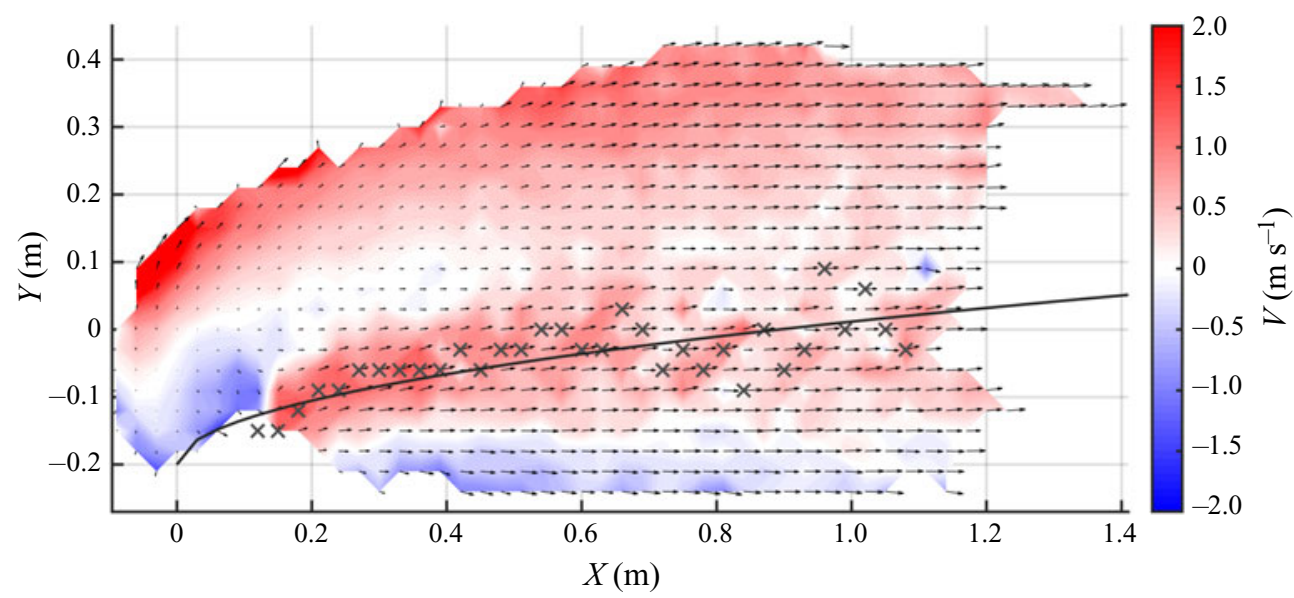

Figure 18. Contours of spanwise velocity $V$ at the plane $Z=0$, for experiments with small snow particles at $U_{\infty}=6.58 \pm 0.45 \mathrm{~ms}^{-1}$. Grey crosses represent the identified cross-flow jet centreline from the data, and the black curve shows the resulting fit according to the profile given by Hasselbrink \& Mungal (2001). Negative velocities upstream of $X \lesssim 0.1 \mathrm{~m}$ were likely spurious, as particles in this region were clumped together and thus hard to identify accurately.

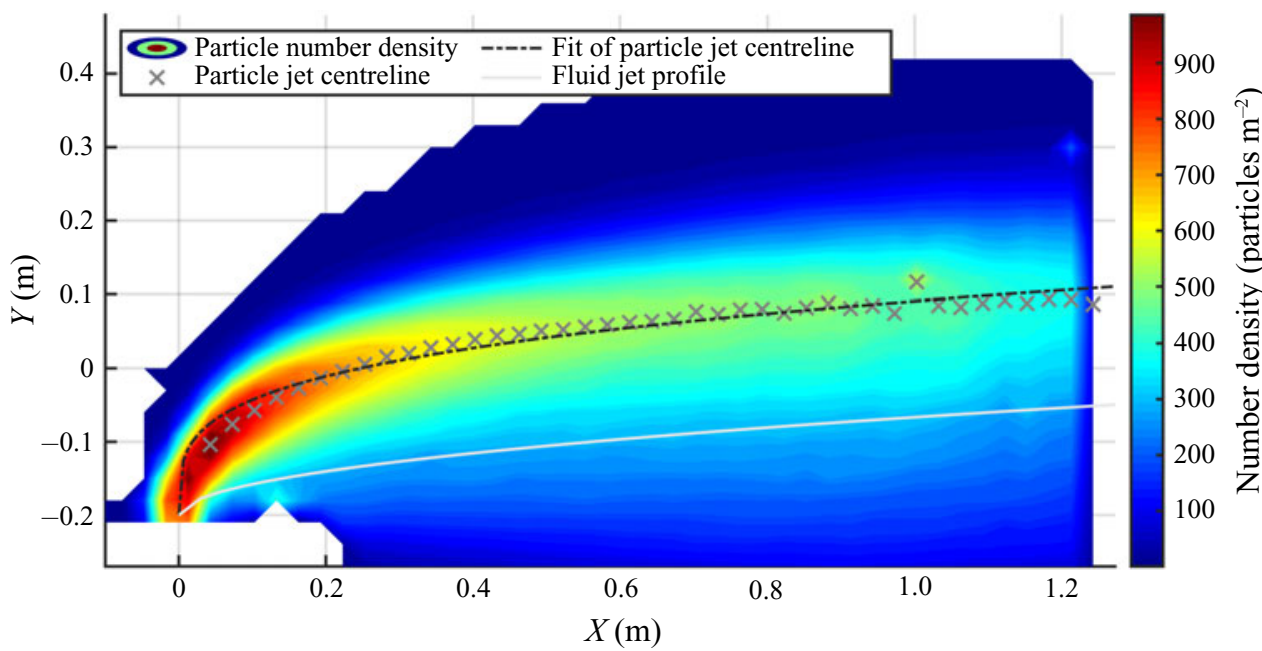

Figure 19. Contours of 2-D particle number density at the plane $Z=0$, for experiments with large snow particles at $U_{\infty}=6.58 \pm 0.45 \mathrm{~ms}^{-1}$. Grey crosses represent the identified particle jet centreline from the data, and the black curve shows the resulting power-law fit, $y(x)=0.291 x^{0.268}-0.2$. The profile of the jet in cross-flow $\left(c_{e} j=0.39, r=0.116\right)$ is given in light grey.

a three-point parabolic fit. The results were then fitted with a two-parameter power-law fit of the form $y(x)=a x^{n}-0.2$, where another parabolic fit was applied along the line $Y=-0.2 \mathrm{~m}$ to fix the coordinate system with respect to the jet orifice.

Given trajectories $y(x)$ for the cross-flow and particle jets and information about the convective velocities along the trajectories, time-dependent profiles $x(t)$ and $y(t)$ could be inferred for the two jets. For the jet in cross-flow, it was assumed that ideal tracer particles would follow the velocity profile derived from the aforementioned similarity solution of 


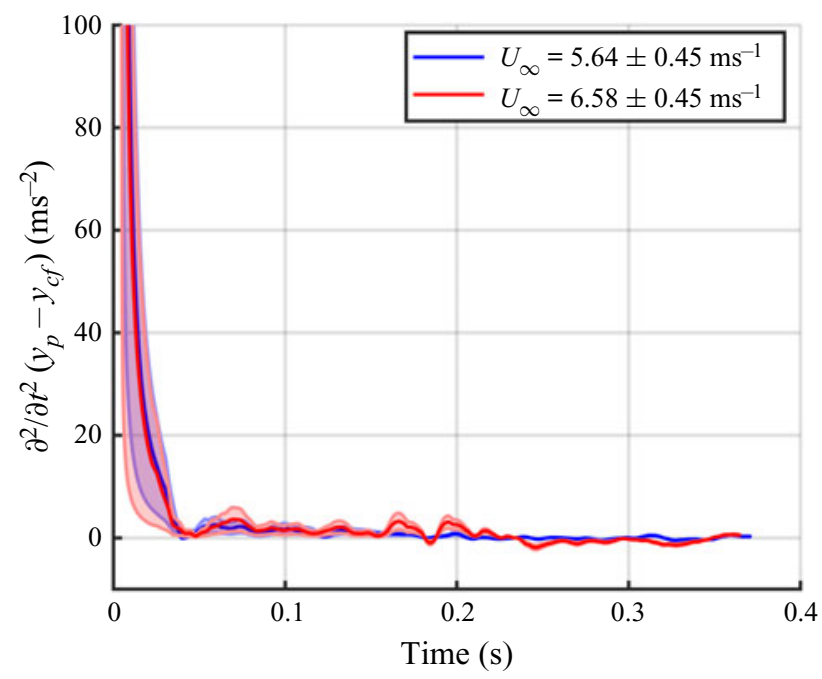

Figure 20. Impulse response in acceleration for the artificial snow particles used in the field experiments, at two free-stream velocities. Oscillations are artefacts of numerical errors from interpolation.

Hasselbrink \& Mungal (2001) as

$$
U(x)=U_{\infty}\left(1-\frac{c_{v j}}{c_{e j}} \frac{d}{x}\right),
$$

where the profile coefficient $c_{v j}$ was taken to be unity. A time history could then be extracted by integrating across point values of $\Delta t=\Delta x / U(x)$. For the particle jet, the streamwise velocity averaged across $Y$ and $Z$ at each streamwise location $X$ was taken to represent the average convective velocity of the particles at that location. This velocity followed a nonlinear relaxation in $X$ that was used to extract an average particle time history. The streamwise coordinates of the cross-flow and particle jets, $x_{c f}(t)$ and $x_{p}(t)$, were computed for a common series of time steps by interpolation. Then, the spanwise coordinates $y_{c f}(t)$ and $y_{p}(t)$ were computed from $x(t)$ using the corresponding fit function for each jet profile.

From the matched time histories of $y_{c f}(t)$ and $y_{p}(t)$, a spanwise error was defined as $\delta(t)=y_{p}(t)-y_{c f}(t)$. This difference represents the deviation of an inertial particle from the background flow. Differentiating once with respect to time yielded the spanwise slip velocity of a particle subjected to a step change in $V$. Differentiating again in time gave the particle's impulse response in acceleration (figure 20). The reliance on numerical differentiation required smooth signals to obtain meaningful results; it is for this reason that fit functions were used instead of measured data to obtain $y(t)$. In addition, a modest smoothing was required on $x(t)$ to prevent interpolation errors from accumulating through numerical differentiation. Error bars were computed from the residuals of the fits for $y_{p}(x)$ and $y_{c f}(x)$. The steady-state value of the profiles was computed as the mean of the latter half of the time history of the signal. The particle-response time scale $\tau_{p}$ was defined as the time at which the profiles of $\partial^{2} \delta / \partial t^{2}$ decayed to within one standard deviation of the converged value. The steady-state values of $\partial \delta / \partial t$ provided an estimation of the slip velocity $V_{s}$ of the particles. Computed particle time scales and slip velocities are given for the four experimental cases in table 2. 


\section{N.J. Wei and others}

$\begin{array}{lcccc}\text { Experimental case } & \text { Small snow 1 } & \text { Small snow 2 } & \text { Large snow 1 } & \text { Large snow 2 } \\ \text { Particle diameter, } d_{p}(\mathrm{~mm}) & 5.9 \pm 1.1 & 5.9 \pm 1.1 & 11.2 \pm 4.2 & 11.2 \pm 4.2 \\ \text { Tunnel speed, } U_{\infty}\left(\mathrm{ms}^{-1}\right) & 5.64 \pm 0.45 & 6.58 \pm 0.45 & 5.64 \pm 0.45 & 6.58 \pm 0.45 \\ \text { Particle time scale, } \tau_{p}(\mathrm{~ms}) & 35.5 \pm 1.2 & 34.4 \pm 2.0 & 39.2 \pm 0.3 & 40.5 \pm 6.7 \\ \text { Slip velocity, } V_{s}\left(\mathrm{~ms}^{-1}\right) & 0.019 \pm 0.013 & 0.025 \pm 0.017 & 0.088 \pm 0.004 & 0.101 \pm 0.030\end{array}$

Table 2. Particle diameters, time scales and estimated slip velocities for the four cases in this experiment. Uncertainties represent one standard deviation from the mean quantities.

\section{A.3. Implications for field experiments}

The small snow particles had slightly shorter time scales and smaller slip velocities than the large snow particles. For both types of particles, the particle-response time scales showed little dependence on the free-stream velocity, while the slip velocities increased modestly with $U_{\infty}$, most noticeably for the large snow particles. This was a consequence of the functional difference between the impulse response in acceleration, which does not depend on the magnitude of the disturbance, and the step response in velocity, which does. The particle-response time scales for the large snow particles were therefore representative of those expected in the field experiments. The relevant flow time scale in the field experiments was $\tau_{f}=D / U_{\infty} \approx 0.18$, resulting in a particle Stokes number of $S k \approx 0.23$. Since $\tau_{p}<\tau_{f}$, it could be assumed that the large snow particles used in the field experiments would respond rapidly enough to resolve the flow structures of interest to this study.

Since the slip velocities showed a tendency to increase with the free-stream velocity, the particle slip velocities in the field experiments were expected to be somewhat larger than those measured in the laboratory experiments. Assuming the particle slip velocity is directly proportional to $U_{\infty}$, a linear extrapolation to the average wind speed at the field site $\left(U_{\infty}=11 \mathrm{~ms}^{-1}\right)$ yields a worst-case particle slip velocity of $V_{s, \max }=0.170 \mathrm{~ms}^{-1}$. The acceleration-impulse condition produced in the wind-tunnel cross-flow configuration was somewhat dramatic compared to conditions encountered by the particles in the field experiments, in which the jet was aligned with the free-stream flow. Therefore, this slip velocity should be treated as an upper bound on the flow fidelity of the particles. Errors due to particle slip in the field experiments were thus estimated to be below $2 \%$ of the free-stream velocity, which was similar to the precision of the 3-D PTV measurement system (cf. appendix B.2).

Lastly, the effect of the snow machines on the flow incident on the wind turbine in the field experiment can be ascertained from the results of the laboratory experiments. The maximum velocity of the flow exiting the snow machine for the large-snow case was $0.993 \pm 0.165 \mathrm{~ms}^{-1}$. Since the snow machines expelled their particles in the streamwise direction in the field experiments, this injection of momentum was minimal compared to the average wind speed. In addition, the particle-response time scales measured in the laboratory suggest that the particles would have equilibrated to the background flow well in advance of their approach to the turbine (of the order of $1 \mathrm{~m}$ from the snow-machine nozzle). Given the turbulent nature of the wind conditions at the field site, the presence of the snow machines was not expected to disrupt the inflow condition to the turbine. These considerations suggested that the effect of the snow machines on the quality and measurement of the flow conditions was negligible. 


\section{Appendix B. Processing procedures}

\section{B.1. Camera calibration}

Calibrations were performed before each turbine was raised into position to facilitate wand motion throughout the measurement volume. One calibration was used for all measurements with the UGE turbine, and a second calibration was used for all measurements with the WPE turbine. A wand, consisting of two 2000-lumen LEDs (XLamp XM-L, Cree Components) spaced $1.15 \mathrm{~m}$ apart, was moved throughout the measurement volume using a reach forklift. A light located in the middle of the wand was flashed to provide a synchronization signal for the cameras. Using the MATLAB tool developed by Theriault et al. (2014), the LED positions and wand lengths were reconstructed in an arbitrarily assigned global coordinate system. The standard deviations of the reconstructed wand lengths from the two calibrations, over all recorded images of the wand as it moved through the measurement domain, were $0.16 \%$ and $0.20 \%$ of the measured wand length. The accuracy of the calibrations was further examined by comparing the calculated and measured distances between cameras, to quantify reprojection errors. The average reprojection errors across all inter-camera distances were $0.74 \pm 0.39 \%$ and $0.83 \pm 0.41 \%$ for the two calibrations. These corresponded to less than $10 \mathrm{~cm}$ in physical space, which was within the error tolerances of the tape-based physical measurements themselves. Lastly, the two calibrations were compared by applying both calibrations to the videos of the first calibration. After matching the two coordinate systems using principal-component analysis, the average distance between corresponding LED positions in the two calibrations was $1.34 \mathrm{~cm}$. This corresponded to a difference on the order of 1 pixel in the camera images, suggesting that the difference between the two calibrations was negligible. Still, for the sake of consistency, the first calibration was used for all of the experiments with the UGE turbine, while the second calibration was used for all experiments with the WPE turbine. As a final check on the validity of the calibrations, images in which the turbines were present in the camera views were used to reconstruct the spans of the turbines. Several points along the top and bottom of each turbine were selected in several camera views, and an elliptical fit of these points identified points lying on the central axis of the turbine. Triangulating the positions of these points in 3-D space (using the methods outlined later in $\S 2.4$ ) yielded turbine spans that differed by $0.21 \%$ and $0.32 \%$, respectively, from the actual values. Overall, these analyses demonstrated that both calibrations were accurate and consistent in their 3-D reconstruction of objects in the measurement domain.

\section{B.2. Three-dimensional PTV processing and statistical convergence}

To obtain accurate time-averaged velocity fields from the raw camera images, a series of processing steps were undertaken. Firstly, for every individual data set, the images from each camera were averaged to produce a background image, which was then subtracted from each image to remove glare and stationary structures. Then, the turbine and support structures visible in each frame were masked using an intensity threshold and object-detection routine. Through this combination of background subtraction and masking, the artificial snow particles in each image were isolated. The results of these steps were checked manually for each data set to confirm that the correct regions of the flow field were isolated.

To compute the temporal offset between images from different cameras, the signal of the synchronization light on the turbine tower was tracked in each image. The mean of the 


\section{N.J. Wei and others}

pixel intensities in a small region containing the synchronization light was computed for every frame in each set of camera images. A custom edge-finding routine was then used to locate the frames in each camera view in which the light was switched on. The six sets of images were temporally aligned according to these reference frames, and the subset of time instances that were represented in all six camera views was selected for the particle-identification step.

To identify particles in this set of images, intensity thresholds were chosen for every camera in each data set. These thresholds were used to binarize the images, and were set manually by iterating on a subset of the images in a data set, so that approximately 200 particle candidates would be identified in each image. This target number of particles was found to be sufficiently high to remain sensitive to poorly illuminated particles, while low enough to prevent false positives from obfuscating actual particles. Pixel coordinates for each particle candidate were determined by computing its centroid, which was more robust to the non-spherical nature of the particles than other measures. These identified coordinates in 2-D image space were then triangulated onto 3-D global coordinates based on the calibrations outlined in $\S 2.2$, using epipolar geometry (Hartley \& Zisserman 2003). To reduce the number of ghost particles (non-physical artefacts from ambiguities in epipolar geometry) detected by this triangulation approach, it was required that a particle appear in at least three camera views in order for it to be triangulated into 3-D space (Elsinga et al. 2010). Particle trajectories and velocities were then numerically computed from these spatial coordinates for all time instances in the data set using a multi-frame predictive-tracking algorithm developed by Ouellette et al. (2006) and Xu (2008). The velocity fields for three data sets with different snow-machine heights were then combined into a single unstructured 3-D volume of three-component velocity vectors. This volume was rotated into the coordinate system given in figure 4 from the arbitrary one assigned by the calibrations using the axis of the turbine and the tips of the snow-machine towers as references. The result of this procedure was a collection of velocity vectors distributed throughout the measurement domain, representing instantaneous velocity measurements at various time instances during the experiment.

The unstructured velocity vectors were then interpolated onto a grid of cubic voxels to produce a single time-averaged velocity field. As the choice of the voxel size influenced both the resolution and statistical convergence of the measured velocity field, statistical analyses were carried out to inform this selection. Firstly, the effect of the number of vectors per voxel on statistical convergence was ascertained. For this analysis, the first case of the helical-bladed UGE turbine was selected. Bootstrap sampling was employed to obtain better estimates of the statistics of the population (Efron 1979). For a voxel with a side length of $25 \mathrm{~cm}$ located upstream of the turbine, 9000 bootstrap samples of $N$ vectors were taken, and the mean and standard deviation of the velocity magnitude were computed for each sample. This process was repeated for values of $N$ from 1 to the total number of vectors in that voxel. The standard deviation of these bootstrapped means represented the uncertainty due to computing an average from samples of the entire population. This uncertainty decreased with increasing $N$, and dropped below $5 \%$ of the average velocity magnitude of all samples in the voxel, $|\boldsymbol{U}|$, at $N \approx 25$ (figure $21 a$ ). The uncertainty fell below $2 \%$ at $N \approx 150$. A similar conclusion regarding the value of $N$ was drawn from the average of the bootstrapped standard deviations for various values of $N$ (figure $21 b$ ). Hence, subsequent analyses sought to include 25 vectors per voxel, where possible.

Given that the distribution of particle traces in the domain was not uniform, a series of voxel sizes was tested on the same data set used above to determine a voxel size that balanced spatial resolution and the target number of vectors per voxel. Voxels of various sizes were used to discretize the domain, and voxels that contained at least three vectors 

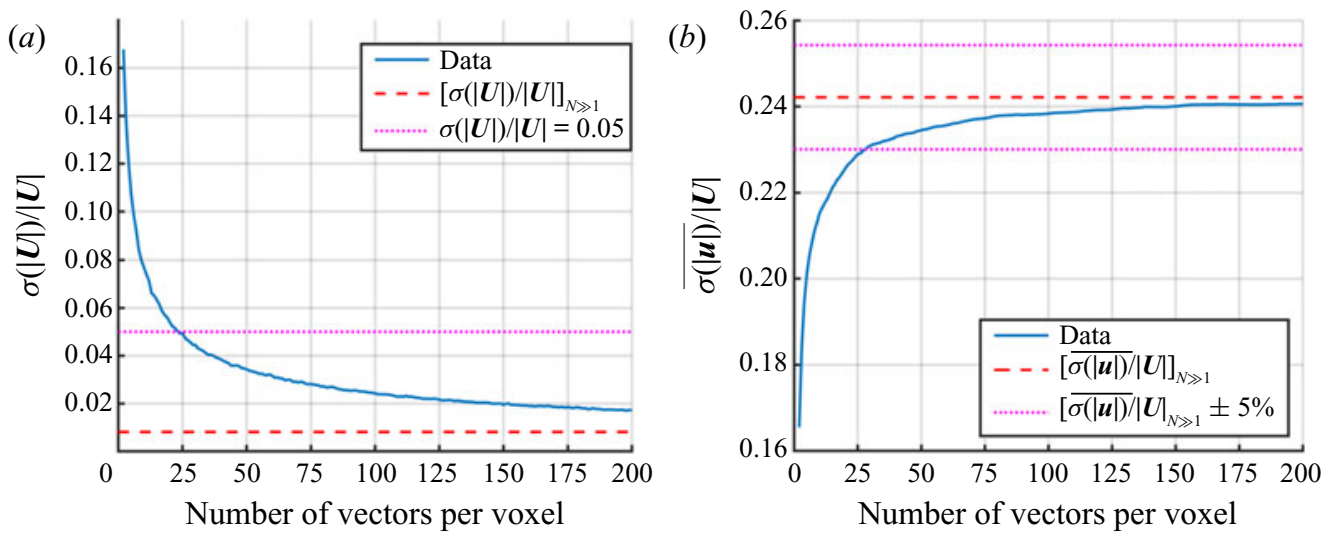

Figure 21. Statistical analysis of vectors contained in a $25-\mathrm{cm}$ cubic voxel, located $1.5 \mathrm{D}$ upstream of the UGE turbine. The variations of the $(a)$ standard deviation of bootstrapped means and $(b)$ mean of bootstrapped standard deviations of the velocity magnitude are shown against the number of vectors taken in each sample. In both figures, the converged value of each measure for $N>>1$ is shown as a red dashed line, while bounds for acceptable convergence are given as dotted magenta lines. $N \gtrsim 25$ yields convergence within $5 \%$, while $N \gtrsim 150$ yields convergence within $2 \%$.

were counted toward the total number of voxels in each discretization. The fraction of these voxels that contained at least 25 vectors began to converge around a grid dimension of $30 \mathrm{~cm}$ (figure $22 a$ ). A grid dimension of $25 \mathrm{~cm}$ resulted in over $50 \%$ of voxels having at least 25 vectors (5\% precision, according to figure $21 a$ ), as well as over $20 \%$ having at least 150 vectors ( $2 \%$ precision). In a volume encapsulating the turbine wake (bounded by $X / D>\frac{1}{2},|Y / D| \leq \frac{3}{2}$ and $\left.|Z / D| \leq \frac{3}{2}\right), 87 \%$ of voxels had at least 25 vectors, and $58 \%$ of voxels had at least 150 vectors. Therefore, the level of precision of the results presented in this work, which focus on this wake volume, was comfortably below $5 \%$ in the area of interest for voxels of this size. In addition, the standard deviation of the bootstrapped means for all vectors within a voxel of a given size dropped below $1 \%$ of $|\boldsymbol{U}|$ for a grid dimension of $25 \mathrm{~cm}$ (figure $22 b$ ). This indicated very good best-case statistical convergence for voxels of at least this size.

In light of these results, a voxel size of $25 \mathrm{~cm}$ was used to discretize the domain, serving as a good compromise between grid resolution and statistical convergence. At least half of all voxels in the measurement volume had standard deviations in the velocity magnitude below $5 \%$, with $20 \%$ of these having a measurement precision below $2 \%$. In the wake of the turbine, the precision for $87 \%$ of the voxels was below $5 \%$, with close to $60 \%$ of this volume having a precision below $2 \%$. The best-case precision for the most densely populated voxels was below $1 \%$.

Once the velocity vectors had been binned and averaged into $25-\mathrm{cm}$ cubic voxels, a filter developed by Schiavazzi et al. (2014) was employed to enforce a zero-divergence criterion on the vector fields, in keeping with the negligible Mach numbers $(M a<0.05)$ of the experimental conditions. The effect of this filter was to attenuate unphysical deviations in the velocity fields due to spurious particle trajectories, especially near the edges of the domain where velocity vectors were more sparse (cf. figure 23).

From these filtered velocity fields, values for the velocity incident on the turbine and the settling velocity of the artificial snow particles were computed by averaging all velocity vectors at least $1 \mathrm{D}$ upstream of the axis of rotation of the turbine. Compared to the settling velocity of the particles measured in quiescent air, $W_{s}^{0}=0.60 \pm 0.18 \mathrm{~ms}^{-1}$, the 


\section{N.J. Wei and others}
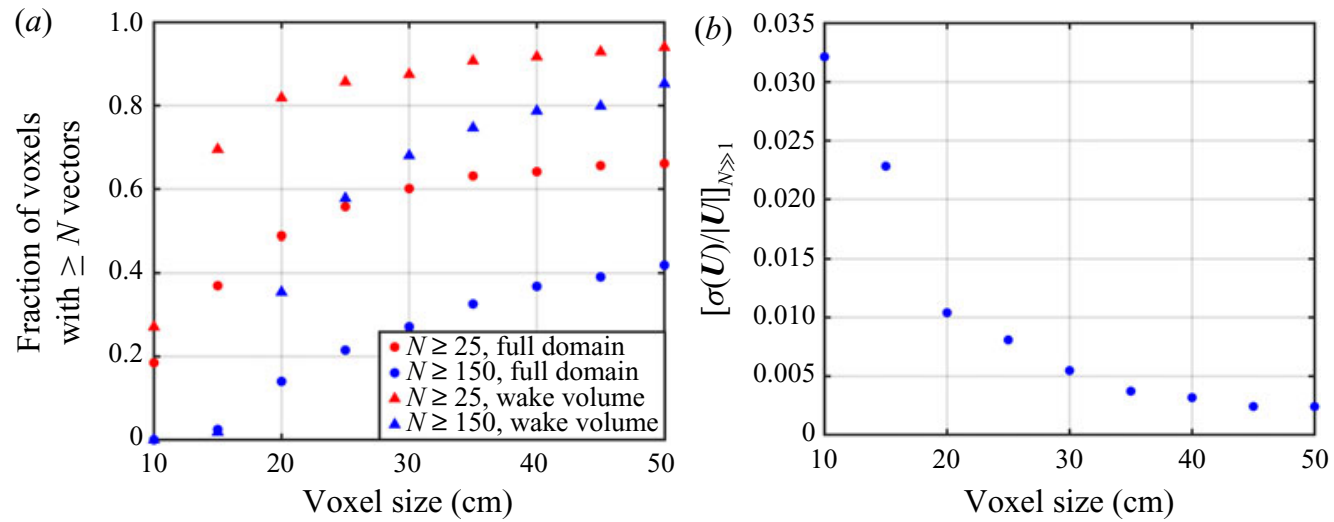

Figure 22. Measures for the determination of an appropriate voxel size for binning and averaging velocity vectors. Panel $(a)$ shows the fraction of voxels containing at least $N=25$ and $N=150$ vectors, representing the number of vectors required for $5 \%$ and $2 \%$ measurement precision, for the entire measurement domain (circles) and the wake region (triangles). Panel $(b)$ shows the standard deviation of bootstrapped means for all vectors in a given voxel, representing the best-case precision possible for a given voxel size. Both measures suggest that a grid dimension of $25 \mathrm{~cm}$ is a good compromise between spatial resolution and statistical convergence.
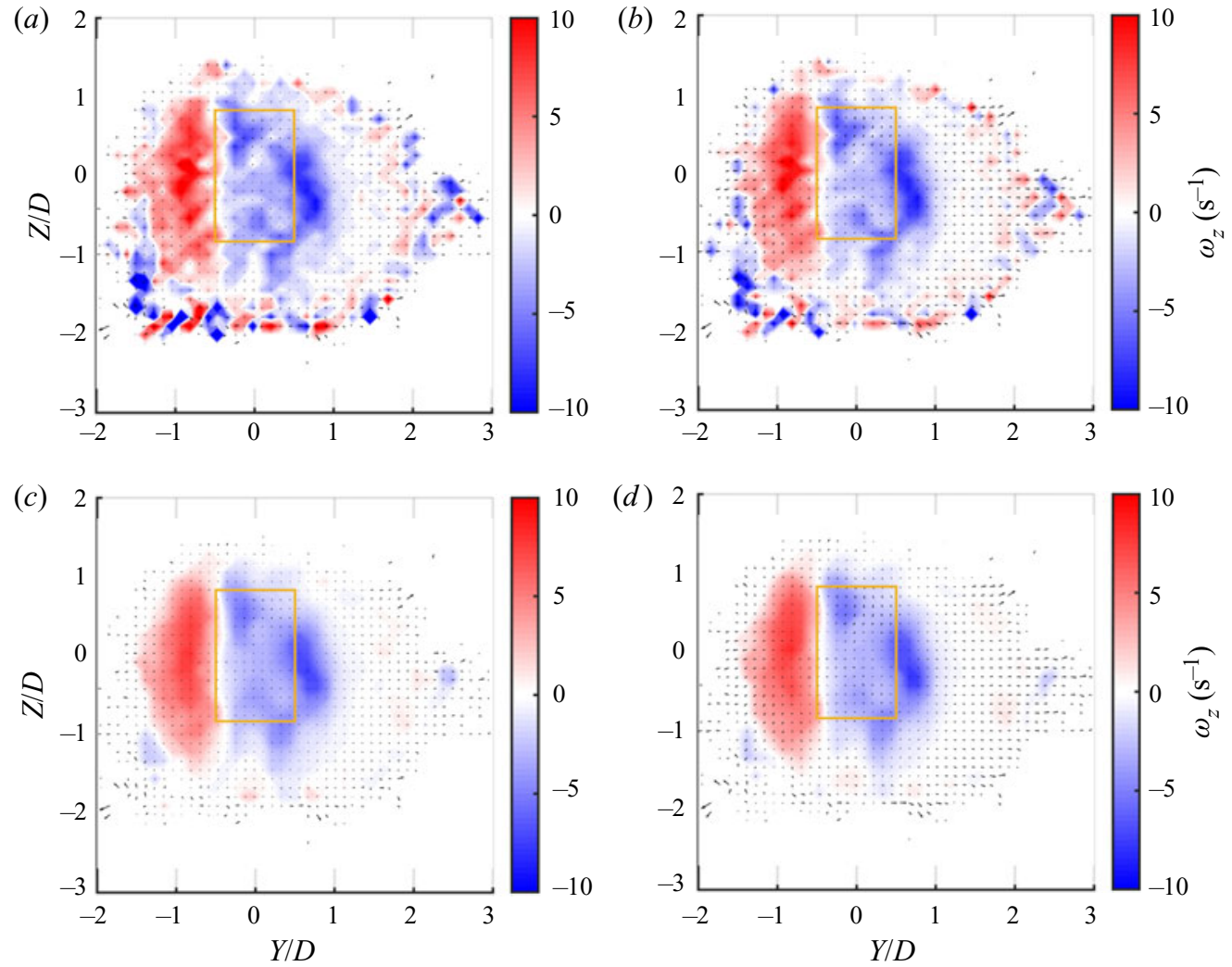

Figure 23. Effects of the two filters applied to the voxel-averaged velocity and vorticity fields, demonstrated on a cross-section of vertical vorticity $\left(\omega_{z}\right)$ at $X / D=1.5$ downstream of the WPE turbine for $\lambda=1.20$. The solenoidal filter affects both the velocity and vorticity readings, while the median filter is only applied to the vorticity field. (a) No filtering; $(b)$ solenoidal filtering only; $(c)$ median filtering only and $(d)$ both solenoidal and median filtering. 
observed settling velocities measured in the prevailing wind conditions at the field site were higher: $W_{s}=0.89 \pm 0.12 \mathrm{~ms}^{-1}$. The discrepancy was likely due to the presence of a slight downward slope in the local topography at the site. According to data collected by Kinzel et al. (2012), the corresponding bias in the vertical velocity was $W \approx-0.22 \mathrm{~ms}^{-1}$, which is consistent with the discrepancy observed in the present data. It is also possible that atmospheric turbulence contributed to the increased settling velocity as well, an effect that has been observed for natural snowflakes (Nemes et al. 2017) and general inertial particles (Petersen et al. 2019) in turbulent conditions. Thus, the average settling velocity measured upstream of the turbine, $W_{s}$, was subtracted from the entire velocity field, so that the time-averaged vertical velocity $W$ induced by the turbine could be isolated.

Fields of vorticity were computed from these velocity fields. Due to the relatively coarse grid size and the error associated with numerical differentiation, a $3 \times 3 \times 3$ median filter was applied to all fields involving velocity derivatives. This was mainly employed to remove unphysical results from numerical differentiation near the edges of the measurement domain, but also had a modest smoothing effect on the vortical structures present in the wake.

To demonstrate the effects of the filters applied to the velocity and vorticity fields, a single planar slice at $X / D=1.5$ was isolated from the WPE turbine data at $\lambda=1.20$, and the vertical vorticity component $\omega_{z}$ was plotted in figure 23 . The unfiltered vorticity fields were marked by significant noise on the boundaries of the domain (figure 23a). Applying the solenoidal filter to the velocity fields resulted in locally smoothed velocity vectors, corresponding to very slight smoothing in the vorticity fields (figure 23b). By contrast, applying the $3 \times 3 \times 3$ median filter to the vorticity fields removed the shot noise from the boundaries of the domain, while smoothing over the large-scale structures (figure 23c). The combination of the two filters resulted in smoothed velocity and vorticity fields that allowed trends in the flow fields to be identified more readily (figure $23 d$ ).

\section{Appendix C. Velocity and vorticity fields}

In this section, salient features of the velocity and vorticity fields in the wake are described and discussed. These results are compared with previous studies in the literature to show that many of the trends observed in laboratory experiments at lower Reynolds numbers still apply for full-scale turbines in field conditions.

\section{C.1. Velocity fields}

Velocity fields for the time-averaged streamwise-velocity component $U$ on three orthogonal planar cross-sections are given for the UGE and WPE turbines, respectively, each at two tip-speed ratios, in figures 24 and 25 . The velocity deficit in the wakes were slightly more pronounced in the higher- $\lambda$ cases, and the wake regions were shifted slightly toward the negative spanwise direction due to the rotation of the turbine. This is consistent with the wake trends observed by Parker \& Leftwich (2016). The magnitude of the velocity deficit was significantly larger for the WPE turbine, consistent with its higher solidity. These flow fields qualitatively paralleled those reported by Araya et al. (2017), which were recorded in a water channel at a significantly lower Reynolds number $\left(R e_{D}=8 \times 10^{4}\right)$. This supports the inference of Parker \& Leftwich (2016) that the general shape of the turbine wake is not very sensitive to Reynolds number in this parameter range, despite the fact that the coefficients of power only converge for $R e_{D} \gtrsim 1.5 \times 10^{6}$ (Miller et al. 2018a).

Velocity fields for the time-averaged spanwise-velocity component $V$, also taken at the mid-span of the turbines, are shown in figures 26(a) and 26(b) for the UGE and WPE 


\section{N.J. Wei and others}
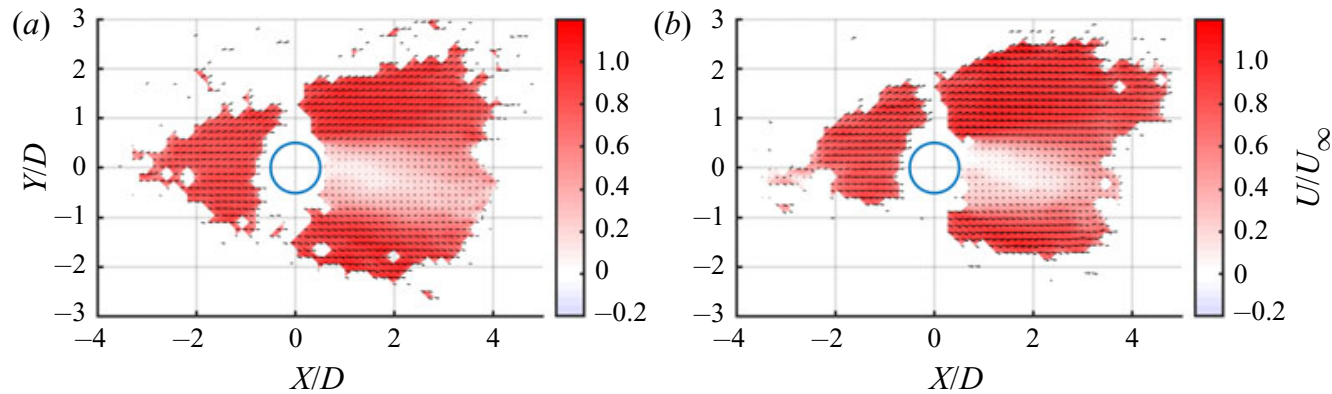

Figure 24. Time-averaged planar fields of the streamwise velocity $U$ for the UGE turbine at $(a) \lambda=1.19$ and (b) $\lambda=1.40$, taken at $Z / D=0$. The differences in the shape of the wake between the two tip-speed ratios are minor.
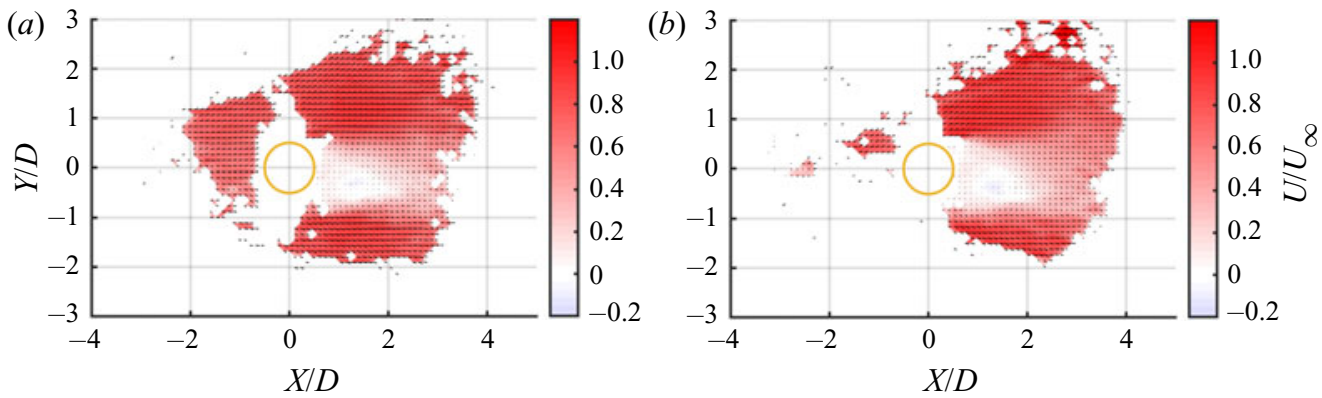

Figure 25. Time-averaged planar fields of the streamwise velocity $U$ for the WPE turbine at $(a) \lambda=0.96$ and (b) $\lambda=1.20$, taken at $Z / D=0$. As in figure 24 , the differences in the wake between these two tip-speed ratios are minor.
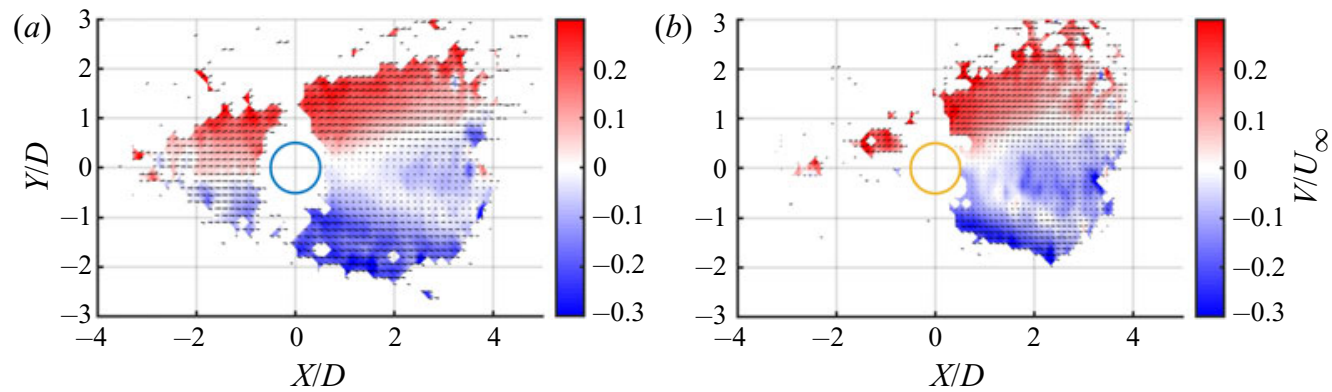

Figure 26. Time-averaged planar fields of the spanwise velocity $V$ for $(a)$ the UGE turbine at $\lambda=1.19$ and (b) the WPE turbine at $\lambda=1.20$, taken at $Z / D=0$. The $\mathrm{V}$-shaped region of negative spanwise velocity downstream of the turbines is more prominent for the WPE turbine, which has a higher solidity.

turbines at $\lambda=1.2$. A clear upstream bifurcation of the flow due to the presence of the turbine was visible in figure 26(a), and a negatively skewed velocity field, representing spanwise flow induced by the rotation of the turbine, was present downstream of the turbine in both cases. This region grew more rapidly in thickness behind the WPE turbine than behind the UGE turbine, again likely due to its higher solidity and correspondingly stronger flow induction. 
Near-wake structure of vertical-axis wind turbines

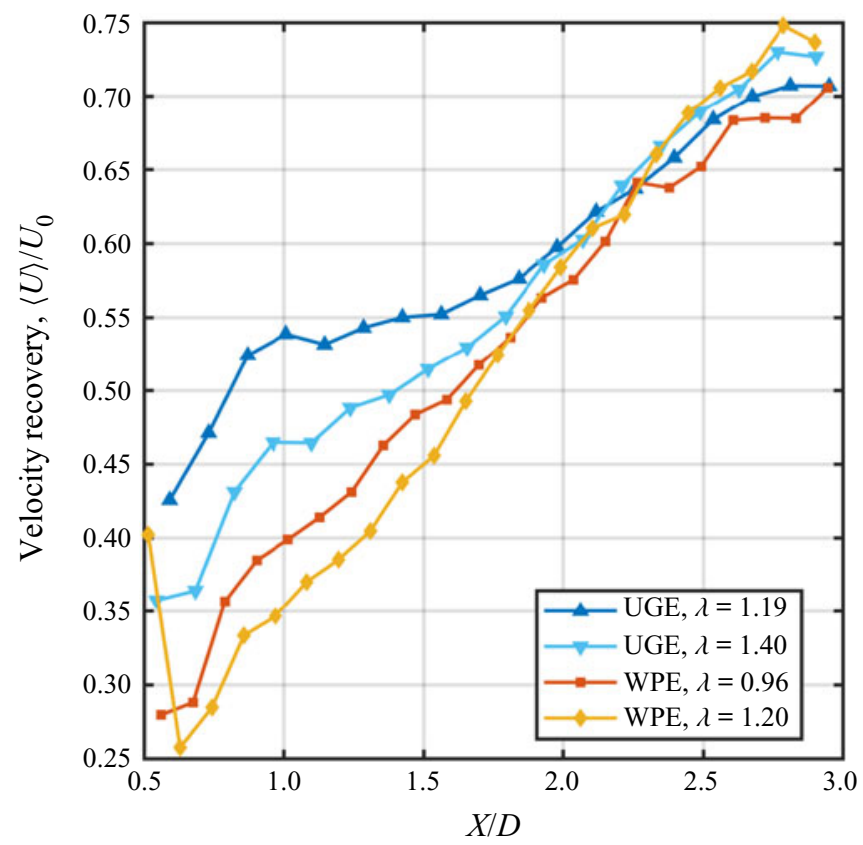

Figure 27. Profiles of $\langle U\rangle / U_{0}$ versus distance downstream of the turbine for all four experimental cases. Here, angle brackets denote spatial averages across $Y Z$-sections of the wake, and $U_{0}$ represents the velocity directly upstream of the turbine. Profile discrepancies corresponding to differences in $\lambda$ and $\sigma$ are present in the near wake $(X / D \lesssim 2)$, whereas the wake recovery in the far wake appears to be more uniform.

The wake recovery of the two turbines also followed previously observed trends in the literature (cf. Ryan et al. 2016; Araya et al. 2017). The wake recovery was quantified by thresholding planar slices of the streamwise velocity component at various streamwise positions downstream of the turbine by the average upstream flow velocity incident on the turbine, $U_{0}$. For each slice, this thresholding procedure divided the wake region, defined as the region of flow where the local streamwise velocity component was less than $U_{\infty}$, from the surrounding free-stream region. The average velocity within the wake region, $\langle U\rangle$, was taken for each streamwise slice, and was plotted against streamwise distance (figure 27). The analysis of these wake-recovery profiles has been undertaken comprehensively by Araya et al. (2017), and the trends found in these experiments show good agreement with their findings. The minimum value of $\langle U\rangle / U_{0}$ was observed to decrease with increasing $\lambda$ and increasing $\sigma$. These differences were most prominent in the near wake $(X / D \lesssim 2)$, in which vortex shedding from the turbine blades is most significant (Tescione et al. 2014; Parker \& Leftwich 2016; Araya et al. 2017). The streamwise extent of the measurement domain was not large enough to observe the full transition to bluff-body wake dynamics described by Araya et al. (2017), but the presently measured wake profiles exhibit similarity to that previous work within the domain of present interest.

\section{C.2. Vorticity fields}

For the sake of completeness, streamwise slices of the spanwise vorticity $\omega_{y}$ are provided in figures 28 and 29. The time-averaged vortical structures visible in these plots represented tip vortices shed from the turbine blades (Tescione et al. 2014). These structures did not 


\section{N.J. Wei and others}

(a)

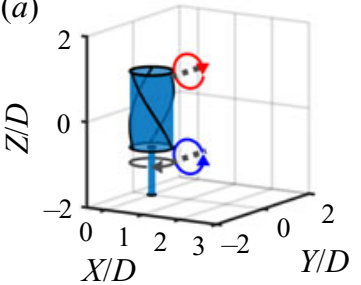

(b)

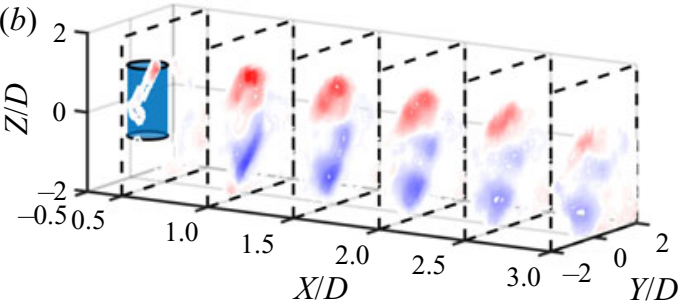

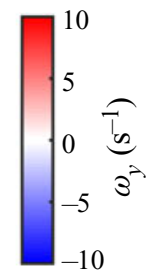
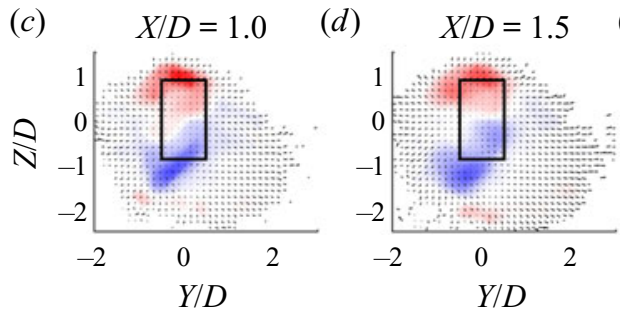

(e)

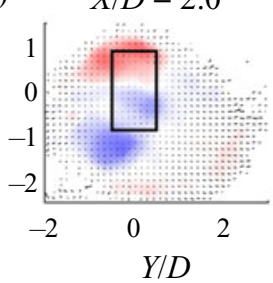

(f) $\quad X / D=2.5$

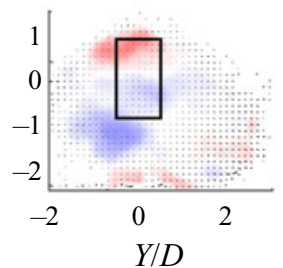

Figure 28. Streamwise slices of the spanwise vorticity $\omega_{y}$ downstream of the UGE turbine for $\lambda=1.19$. These structures are products of vortex shedding from the tips of the turbine blades (Tescione et al. 2014). Note that the $X$-axis is stretched on $0.5 \leq X / D \leq 3$.

(a)

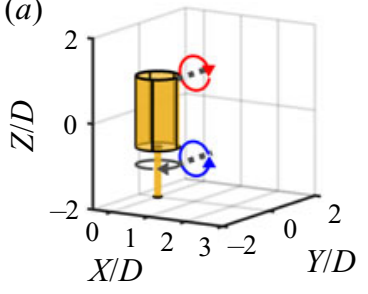

(b)

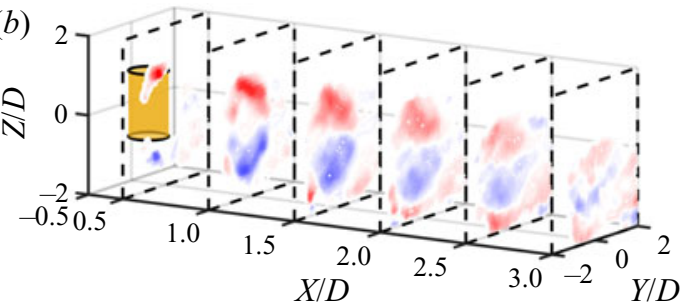

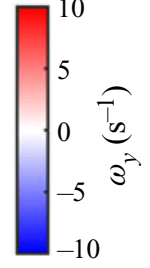

(e)

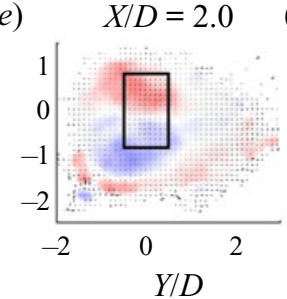

(f) $\quad X / D=2.5$

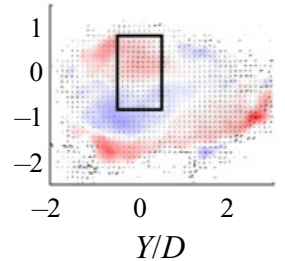

Figure 29. Streamwise slices of the spanwise vorticity $\omega_{y}$ downstream of the WPE turbine for $\lambda=1.20$. These structures are not significantly different from those shown in figure 28.

display a strong degree of asymmetry, suggesting that they were relatively unaffected by the tilted-wake behaviour observed in the vortical structures in $\omega_{x}$ and $\omega_{z}$.

\section{REFERENCES}

ABKAR, M. 2018 Impact of subgrid-scale modeling in actuator-line based large-eddy simulation of vertical-axis wind turbine wakes. Atmosphere 9 (7), 257.

ABKAR, M. \& DABIRI, J.O. 2017 Self-similarity and flow characteristics of vertical-axis wind turbine wakes: an LES study. J. Turbul. 18 (4), 373-389.

Abraham, A. \& Hong, J. 2020 Dynamic wake modulation induced by utility-scale wind turbine operation. Appl. Energy 257, 114003. 
Ahmadi-Baloutaki, M., CarRiveau, R. \& Ting, D.S.-K. 2016 A wind tunnel study on the aerodynamic interaction of vertical axis wind turbines in array configurations. Renew. Energy 96, 904-913.

Aliferis, A.D., Jessen, M.S., Bracchi, T. \& Hearst, R.J. 2019 Performance and wake of a Savonius vertical-axis wind turbine under different incoming conditions. Wind Energy 22 (9), 1260-1273.

Aliseda, A., Cartellier, A., Hainaux, F. \& Lasheras, J.C. 2002 Effect of preferential concentration on the settling velocity of heavy particles in homogeneous isotropic turbulence. J. Fluid Mech. 468, 77-105.

Araya, D.B., Colonius, T. \& Dabiri, J.O. 2017 Transition to bluff-body dynamics in the wake of vertical-axis wind turbines. J. Fluid Mech. 813, 346-381.

ARMS, R.J. \& HAMA, F.R. 1965 Localized-induction concept on a curved vortex and motion of an elliptic vortex ring. Phys. Fluids 8 (4), 553-559.

Barthelmie, R.J., Frandsen, S.T., Nielsen, M.N., Pryor, S.C., Rethore, P.-E. \& Jørgensen, H.E. 2007 Modelling and measurements of power losses and turbulence intensity in wind turbine wakes at Middelgrunden offshore wind farm. Wind Energy 10 (6), 517-528.

BARTHELmie, R.J. \& JenSEn, L.E. 2010 Evaluation of wind farm efficiency and wind turbine wakes at the Nysted offshore wind farm. Wind Energy 13 (6), 573-586.

Barthelmie, R.J., Pryor, S.C., Frandsen, S.T., Hansen, K.S., Schepers, J.G., Rados, K., Schlez, W., Neubert, A., Jensen, L.E. \& Neckelmann, S. 2010 Quantifying the impact of wind turbine wakes on power output at offshore wind farms. J. Atmos. Ocean. Technol. 27, 1302-1317.

Bartl, J., Mühle, F., Schottler, J., SÆtran, L., Peinke, J., Adaramola, M. \& Hölling, M. 2018 Wind tunnel experiments on wind turbine wakes in yaw: effects of inflow turbulence and shear. Wind Energy Sci. 3 (1), 329-343.

Bastankhah, M. \& Porté-Agel, F. 2016 Experimental and theoretical study of wind turbine wakes in yawed conditions. J. Fluid Mech. 806, 506-541.

Bastankhah, M. \& Porté-Agel, F. 2017 Wind tunnel study of the wind turbine interaction with a boundary-layer flow: upwind region, turbine performance, and wake region. Phys. Fluids 29 (6), 065105.

Battisti, L., Zanne, L., Dell’Anna, S., Dossena, V., Persico, G. \& Paradiso, B. 2011 Aerodynamic measurements on a vertical axis wind turbine in a large scale wind tunnel. J. Energy Resour. Technol. 133 (3), 031201.

BetZ, A. 1920 Das Maximum der theoretisch möglichen Ausnützung des Windes durch Windmotoren. Zeitschrift für das gesamte Turbinenwesen 26, 307-309.

BöHM, H.P. 1988 A general equation for the terminal fall speed of solid hydrometeors. J. Atmos. Sci. 46 (15), 2419-2427.

Bossuyt, J., Howland, M.F., Meneveau, C. \& Meyers, J. 2016 Measurement of unsteady loading and power output variability in a micro wind farm model in a wind tunnel. Exp. Fluids 58 (1), 1.

Boulanger, N., Meunier, P. \& Dizès, S.L. 2008 Tilt-induced instability of a stratified vortex. J. Fluid Mech. 596, 1-20.

Bourgoin, M. \& XU, H. 2014 Focus on dynamics of particles in turbulence. New J. Phys. 16 (8), 085010.

BRemseth, J. \& Duraisamy, K. 2016 Computational analysis of vertical axis wind turbine arrays. Theor. Comput. Fluid Dyn. 30 (5), 387-401.

Brochier, G., Fraunie, P. \& BeguierJ, C. 1986 Water channel experiments of dynamic stall on Darrieus wind turbine blades. J. Propul. Power 2 (5), 445-449.

Brownstein, I.D., KinZel, M. \& DabiRi, J.O. 2016 Performance enhancement of downstream vertical-axis wind turbines. J. Renew. Sustain. Energy 8 (5), 053306.

BRownSteIn, I.D., WEI, N.J. \& DABIRI, J.O. 2019 Aerodynamically interacting vertical-axis wind turbines: performance enhancement and three-dimensional flow. Energies 12 (14), 2724.

Buchner, A.-J., Lohry, M.W., Martinelli, L., Soria, J. \& Smits, A.J. 2015 Dynamic stall in vertical axis wind turbines: comparing experiments and computations. J. Wind Engng Ind. Aerodyn. 146, $163-171$.

Buchner, A.-J., Soria, J., Honnery, D. \& Smits, A.J. 2018 Dynamic stall in vertical axis wind turbines: scaling and topological considerations. J. Fluid Mech. 841, 746-766.

Cal, R.B., Lebrón, J., Castillo, L., Kang, H.S. \& Meneveau, C. 2010 Experimental study of the horizontally averaged flow structure in a model wind-turbine array boundary layer. J. Renew. Sustain. Energy 2 (1), 013106.

Calaf, M., Meneveau, C. \& Meyers, J. 2010 Large eddy simulation study of fully developed wind-turbine array boundary layers. Phys. Fluids 22 (1), 015110.

Caridi, G.C.A., Ragni, D., Sciacchitano, A. \& Scarano, F. 2016 HFSB-seeding for large-scale tomographic PIV in wind tunnels. Exp. Fluids 57 (12), 190.

CHAMORRO, L.P. \& Porté-Agel, F. 2009 A wind-tunnel investigation of wind-turbine wakes: boundary layer turbulence effects. Boundary-Layer Meteorol. 132 (1), 129-149. 


\section{N.J. Wei and others}

Cheng, Q., LiU, X., Ji, H.S., Kim, K.C. \& YAnG, B. 2017 Aerodynamic analysis of a helical vertical axis wind turbine. Energies 10 (4), 575.

Craig, A.E., DAbiri, J.O. \& KosefF, J.R. 2016 A kinematic description of the key flow characteristics in an array of finite-height rotating cylinders. J. Fluids Engng 138 (7), 070906.

DABIRI, J.O. 2011 Potential order-of-magnitude enhancement of wind farm power density via counter-rotating vertical-axis wind turbine arrays. J. Renew. Sustain. Energy 3, 043104.

Dunne, R. \& MCKeOn, B.J. 2015 Dynamic stall on a pitching and surging airfoil. Exp. Fluids 56 (8), 157.

EFron, B. 1979 Bootstrap methods: another look at the jackknife. Ann. Stat. 7 (1), 1-26.

Elsinga, G.E., Westerweel, J., Scarano, F. \& Novara, M. 2010 On the velocity of ghost particles and the bias errors in tomographic-PIV. Exp. Fluids 50 (4), 825-838.

Ferreira, C.S., VAn Kuik, G., VAn Bussel, G. \& SCARAno, F. 2009 Visualization by PIV of dynamic stall on a vertical axis wind turbine. Exp. Fluids 46, 97-108.

Fleming, P., Annoni, J., Churchfield, M., Martinez-Tossas, L.A., Gruchalla, K., Lawson, M. \& MORIARTY, P. 2018 A simulation study demonstrating the importance of large-scale trailing vortices in wake steering. Wind Energy Sci. 3 (1), 243-255.

Fleming, P., Annoni, J., Scholbrock, A., Quon, E., Dana, S., Schreck, S., RaAch, S., Haizmann, F. \& SCHLipf, D. 2017 Full-scale field test of wake steering. J. Phys.: Conf. Ser. 854, 012013.

FLEMING, P., et al. 2019 Initial results from a field campaign of wake steering applied at a commercial wind farm - Part 1. Wind Energy Sci. 4 (2), 273-285.

Fric, T.F. \& Roshko, A. 1994 Vortical structure in the wake of a transverse jet. J. Fluid Mech. 279, 1-47.

Gopalan, S., Abraham, B.M. \& Katz, J. 2004 The structure of a jet in cross flow at low velocity ratios. Phys. Fluids 16 (6), 2067-2087.

Gorlov, A.M. 1995 United States Patent: 5451137 - unidirectional helical reaction turbine operable under reversible fluid flow for power systems. United States Government.

Hama, F.R. \& Nutant, J. 1961 Self-induced velocity on a curved vortex. Phys. Fluids 4 (1), $28-32$.

Han, D., Heo, Y.G., Choi, N.J., Nam, S.H., Choi, K.H. \& Kim, K.C. 2018 Design, fabrication, and performance test of a 100-W helical-blade vertical-axis wind turbine at low tip-speed ratio. Energies 11 (6), 1517.

Hartley, R. \& Zisserman, A. 2003 Multiple View Geometry, 2nd edn. Cambridge University Press.

Hasselbrink, E.F. \& Mungal, M.G. 2001 Transverse jets and jet flames. Part 1. Scaling laws for strong transverse jets. J. Fluid Mech. 443, 1-25.

HaU, E. 2013 Wind Turbines Fundamentals, Technologies, Application, Economics. Springer.

HeZAVEH, S.H. \& Bou-ZEID, E. 2018 Mean kinetic energy replenishment mechanisms in vertical-axis wind turbine farms. Phys. Rev. Fluids 3, 094606.

Hezaveh, S.H., Bou-Zeid, E., Dabiri, J.O., Kinzel, M., Cortina, G. \& Martinelli, L. 2018 Increasing the power production of vertical-axis wind-turbine farms using synergistic clustering. Boundary-Layer Meteorol. 169 (2), 275-296.

Hofemann, C., Ferreira, C.S., Dixon, K., Van Bussel, G., Van Kuik, G. \& Scarano, F. 2008 3D Stereo PIV study of tip vortex evolution on a VAWT. In The Proceedings of the European Wind Energy Conference and exhibition EWEC, Brussels, 1-8 2008. European Wind Energy Association (EWEA).

Hong, J., Toloui, M., Chamorro, L.P., Guala, M., Howard, K., Riley, S., Tucker, J. \& Sotiropoulos, F. 2014 Natural snowfall reveals large-scale flow structures in the wake of a 2.5-MW wind turbine. Nat. Commun. 5, 4216.

Howland, M.F., Bossuyt, J., Martínez-Tossas, L.A., Meyers, J. \& Meneveau, C. 2016 Wake structure in actuator disk models of wind turbines in yaw under uniform inflow conditions. J. Renew. Sustain. Energy 8 (4), 043301.

Howland, M.F., LelE, S.K. \& DABIRI, J.O. 2019 Wind farm power optimization through wake steering. Proc. Natl Acad. Sci. USA 116 (29), 14495-14500.

Ilak, M., Schlatter, P., BAgheri, S. \& Henningson, D.S. 2012 Bifurcation and stability analysis of a jet in cross-flow: onset of global instability at a low velocity ratio. J. Fluid Mech. 696, 94-121.

IREland, P.J., BRAGG, A.D. \& Collins, L.R. 2016 The effect of Reynolds number on inertial particle dynamics in isotropic turbulence. Part 2. Simulations with gravitational effects. J. Fluid Mech. 796, 659-711.

JACOBSON, M.Z. \& ARCHER, C.L. 2012 Saturation wind power potential and its implications for wind energy. Proc. Natl Acad. Sci. USA 109 (39), 15679-15684.

Karagozian, A.R. 2014 The jet in crossflow. Phys. Fluids 26 (10), 101303.

Kinzel, M., ARAYA, D.B. \& DABIRI, J.O. 2015 Turbulence in vertical axis wind turbine canopies. Phys. Fluids 27 (11), 115102. 


\section{Near-wake structure of vertical-axis wind turbines}

Kinzel, M., Mulligan, Q. \& DABiRI, J.O. 2012 Energy exchange in an array of vertical-axis wind turbines. J. Turbul. 13, N38.

Klimas, P.C. \& Worstell, M.H. 1981 Effects of blade preset pitch/offset on curved-blade Darrieus vertical axis wind turbine performance. Tech. Rep. SAND-81-1762. Sandia National Labs.

Klotz, L., Gumowski, K. \& Wesfreid, J.E. 2019 Experiments on a jet in a crossflow in the low-velocity-ratio regime. J. Fluid Mech. 863, 386-406.

LeonARD, A. 1985 Computing three-dimensional incompressible flows with vortex elements. Annu. Rev. Fluid Mech. 17 (1), 523-559.

Luzzatto-Fegiz, P. \& CAulfield, C.-C.P. 2018 Entrainment model for fully-developed wind farms: effects of atmospheric stability and an ideal limit for wind farm performance. Phys. Rev. Fluids 3 (9), 093802 .

Mahesh, K. 2013 The interaction of jets with crossflow. Annu. Rev. Fluid Mech. 45 (1), 379-407.

Meneveau, C. 2012 The top-down model of wind farm boundary layers and its applications. J. Turbul. $13, \mathrm{~N} 7$.

Meyers, J. \& Meneveau, C. 2012 Optimal turbine spacing in fully developed wind farm boundary layers. Wind Energy 15 (2), 305-317.

Miller, M.A., Duvvuri, S., Brownstein, I., Lee, M., Dabiri, J.O. \& Hultmark, M. $2018 a$ Vertical-axis wind turbine experiments at full dynamic similarity. J. Fluid Mech. 844, 707-720.

Miller, M.A., Duvvuri, S., Kelly, W.D. \& Hultmark, M. $2018 b$ Rotor solidity effects on the performance of vertical-axis wind turbines at high Reynolds numbers. In The Science of Making Torque from Wind (TORQUE), vol. 1037. IOP Conference Series.

Moin, P., LeOnARD, A. \& KiM, J. 1986 Evolution of a curved vortex filament into a vortex ring. Phys. Fluids 29 (4), 955-963.

Möllerström, E., Gipe, P., Beurskens, J. \& Ottermo, F. 2019 A historical review of vertical axis wind turbines rated $100 \mathrm{~kW}$ and above. Renew. Sustain. Energy Rev. 105, 1-13.

Nemes, A., Dasari, T., Hong, J., Guala, M. \& Coletti, F. 2017 Snowflakes in the atmospheric surface layer: observation of particle-turbulence dynamics. J. Fluid Mech. 814, 592-613.

NyGAARD, N.G. \& NEWCOMBE, A.C. 2018 Wake behind an offshore wind farm observed with dual-Doppler radars. J. Phys.: Conf. Ser. 1037, 072008.

Ouellette, N.T., Xu, H. \& Bodenschatz, E. 2006 A quantitative study of three-dimensional Lagrangian particle tracking algorithms. Exp. Fluids 40, 301-313.

Ouro, P., Runge, S., Luo, Q. \& Stoesser, T. 2019 Three-dimensionality of the wake recovery behind a vertical axis turbine. Renew. Energy 133, 1066-1077.

PARker, C.M, ArAyA, D.B. \& LefTwiCh, M.C. 2017 Effect of chord-to-diameter ratio on vertical-axis wind turbine wake development. Exp. Fluids 58, 168.

PARKER, C.M. \& LEFTwiCH, M.C. 2016 The effect of tip speed ratio on a vertical axis wind turbine at high Reynolds numbers. Exp. Fluids 57, 74.

Petersen, A.J., BAKer, L. \& Coletti, F. 2019 Experimental study of inertial particles clustering and settling in homogeneous turbulence. J. Fluid Mech. 864, 925-970.

PosA, A. \& BALARAS, E. 2018 Large eddy simulation of an isolated vertical axis wind turbine. J. Wind Engng Ind. Aerodyn. 172, 139-151.

Posa, A., Parker, C.M., Leftwich, M.C. \& Balaras, E. 2016 Wake structure of a single vertical axis wind turbine. Intl J. Heat Fluid Flow 61, 75-84.

Rajagopalan, R.G., RickerL, T.L. \& Klimas, P.C. 1990 Aerodynamic interference of vertical axis wind turbines. J. Propul. Power 6 (5), 645-653.

Rezaeiha, A., Montazeri, H. \& Blocken, B. 2018 Towards optimal aerodynamic design of vertical axis wind turbines: impact of solidity and number of blades. Energy 165, 1129-1148.

Ricou, F.P. \& SPALDing, D.B. 1961 Measurements of entrainment by axisymmetrical turbulent jets. J. Fluid Mech. 11 (1), 21-32.

Rolin, V. \& Porté-Agel, F. 2015 Wind-tunnel study of the wake behind a vertical axis wind turbine in a boundary layer flow using stereoscopic particle image velocimetry. J. Phys.: Conf. Ser. 625, 012012.

Rolin, V.F.-C. \& PORTÉ-AGEL, F. 2018 Experimental investigation of vertical-axis wind-turbine wakes in boundary layer flow. Renew. Energy 118, 1-13.

Ryan, K.J., Coletti, F., Elkins, C.J., Dabiri, J.O. \& Eaton, J.K. 2016 Three-dimensional flow field around and downstream of a subscale model rotating vertical axis wind turbine. Exp. Fluids 57, 38.

Schiavazzi, D., Coletti, F., Iaccarino, G. \& Eaton, J.K. 2014 A matching pursuit approach to solenoidal filtering of three-dimensional velocity measurements. J. Comput. Phys. 263, 206-221.

Schottler, J., Reinke, N., Hölling, A., Whale, J., Peinke, J. \& Hölling, M. 2017 On the impact of non-Gaussian wind statistics on wind turbines - an experimental approach. Wind Energy Sci. 2 (1), 1-13. 


\section{N.J. Wei and others}

SCHUERICH, F. \& BROWN, R.E. 2011 Effect of dynamic stall on the aerodynamics of vertical-axis wind turbines. AIAA J. 49 (11), 2511-2521.

Shamsodin, S. \& PortÉ-Agel, F. 2014 Large eddy simulation of vertical axis wind turbine wakes. Energies 7 (2), 890-912.

ShAMSOddin, S. \& PorTÉ-AGEL, F. 2016 A large-eddy simulation study of vertical axis wind turbine wakes in the atmospheric boundary layer. Energies 9 (5), 366.

Shapiro, C.R., Gayme, D.F. \& Meneveau, C. 2018 Modelling yawed wind turbine wakes: a lifting line approach. J. Fluid Mech. 841, R1.

Smith, L.R. \& Jones, A.R. 2019 Measurements on a yawed rotor blade pitching in reverse flow. Phys. Rev. Fluids 4 (3), 034703.

Stevens, R.J.A.M. \& Meneveau, C. 2017 Flow structure and turbulence in wind farms. Annu. Rev. Fluid Mech. 49 (1), 311-339.

Su, L.K. \& Mungal, M.G. 2004 Simultaneous measurements of scalar and velocity field evolution in turbulent crossflowing jets. J. Fluid Mech. 513, 1-45.

Tescione, G., Ragni, D., He, C., Ferreira, C.J.S. \& Van Bussel, G.J.W. 2014 Near wake flow analysis of a vertical axis wind turbine by stereoscopic particle image velocimetry. Renew. Energy 70, 47-61.

Theriault, D.H., Fuller, N.W., Jackson, B.E., Bluhm, E., Evangelista, D., Wu, Z., Betke, M. \& HEDRICK, T.L. 2014 A protocol and calibration method for accurate multi-camera field videography. J. Expl Biol. 217, 1843-1848.

Toschi, F. \& Bodenschatz, E. 2009 Lagrangian properties of particles in turbulence. Annu. Rev. Fluid Mech. 41 (1), 375-404.

Vermeer, L.J., Sørensen, J.N. \& Crespo, A. 2003 Wind turbine wake aerodynamics. Prog. Aerosp. Sci. 39 (6), 467-510.

Villeneuve, T., Boudreau, M. \& Dumas, G. 2020 Improving the efficiency and the wake recovery rate of vertical-axis turbines using detached end-plates. Renew. Energy 150, 31-45.

VisBAL, M.R. \& GARMANN, D.J. 2019 Effect of sweep on dynamic stall of a pitching finite-aspect-ratio wing. AIAA J. 57 (8), 3274-3289.

Wong, J.G., LABASTIDE, B.P. \& Rival, D.E. 2017 Flow separation on flapping and rotating profiles with spanwise gradients. Bioinspir. Biomim. 12 (2), 026008.

Xu, H. 2008 Tracking Lagrangian trajectories in position-velocity space. Meas. Sci. Technol. 19, 075105.

XU, H. \& Bodenschatz, E. 2008 Motion of inertial particles with size larger than Kolmogorov scale in turbulent flows. Physica D 237 (14), 2095-2100.

Yang, X., Hong, J., Barone, M. \& Sotiropoulos, F. 2016 Coherent dynamics in the rotor tip shear layer of utility-scale wind turbines. J. Fluid Mech. 804, 90-115.

ZANFORLin, S. \& Nishino, T. 2016 Fluid dynamic mechanisms of enhanced power generation by closely spaced vertical axis wind turbines. Renew. Energy 99, 1213-1226. 